\title{
GLOBAL PROCESSES THAT DETERMINE COSMIC RAY MODULATION
}

\author{
Report of Working Group 1
}

\author{
L. A. FISK ${ }^{1}$ and K.-P. WENZEL ${ }^{2}$ \\ Co-Chairs
}
A. BALOGH ${ }^{3}$, R. A. BURGER ${ }^{4}$, A. C. CUMMINGS $^{5}$, P. EVENSON ${ }^{6}$, B. HEBER $^{7}$, J. R. JOKIPII ${ }^{8}$, M. B. KRAINEV ${ }^{9}$, J. KÓTA ${ }^{8}$, H. KUNOW ${ }^{7}$, J. A. LE ROUX ${ }^{10}$, F. B. MCDONALD ${ }^{10}$, R. B. MCKIBBEN ${ }^{11}$, M. S. POTGIETER ${ }^{4}$, J. A. SIMPSON ${ }^{11}$, C. D. STEENBERG ${ }^{4}$, S. SUESS ${ }^{12}$, W. R. WEBBER ${ }^{13}$, G. WIBBERENZ $^{7}$ and M. ZHANG ${ }^{11}$
Participants

P. FERRANDO ${ }^{14}$, Z. FUJII ${ }^{15}$, J. A. LOCKWOOD ${ }^{16}$, H. MORAAL ${ }^{4}$ and E. C. STONE ${ }^{5}$

Contributing Authors not participating in the Workshop

${ }^{1}$ Dept. of Atmospheric, Oceanic, and Space Sciences, Univ. of Michigan, Ann Arbor, MI 48109, USA

${ }^{2}$ Space Science Department of ESA, ESTEC, NL-2200 AG Noordwijk, The Netherlands

${ }^{3}$ The Blackett Laboratory, Imperial College, London SW7 2BZ, United Kingdom

${ }^{4}$ Potchefstroom University for CHE, Potchefstroom, South Africa

${ }^{5}$ California Institute of Technology, Pasadena, CA 91125, USA

${ }^{6}$ Bartol Research Institute, University of Delaware, Newark, DE 19716, USA

${ }^{7}$ Institut für Experimentelle und Angewandte Physik, Universität Kiel, 24118 Kiel, Germany

${ }^{8}$ University of Arizona, Tucson, AZ 85721, USA

${ }^{9}$ Lebedev Physical Institute, Russian Academy of Sciences, Moscow, 117924, Russia

${ }^{10}$ Institute for Physical Science and Technology, Univ. of Maryland, College Park, MD 20742, USA

${ }^{11}$ Enrico Fermi Institute and Dept. of Physics, The University of Chicago, Chicago, IL 60637, USA

${ }^{12}$ NASA Marshall Space Flight Center/ES82, Huntsville, AL 35812, USA

${ }^{13}$ New Mexico State University, Las Cruces, NM 88003-001, USA

${ }^{14}$ CEA, DSM/DAPNIA/Service d'Astrophysique, CE-Saclay, F-91191 Gif-sur-Yvette Cedex, France

${ }^{15}$ Solar-Terrestrial Environment Laboratory, Nagoya University, Nagoya, Japan

${ }^{16}$ Space Science Center, University of New Hampshire, Durham, NH 03842, USA

\begin{abstract}
The global processes that determine cosmic ray modulation are reviewed. The essential elements of the theory which describes cosmic ray behavior in the heliosphere are summarized, and a series of discussions is presented which compare the expectations of this theory with observations of the spatial and temporal behavior of both galactic cosmic rays and the anomalous component; the behavior of cosmic ray electrons and ions; and the 26-day variations in cosmic rays as a function of heliographic latitude. The general conclusion is that the current theory is essentially correct. There is clear evidence, in solar minimum conditions, that the cosmic rays and the anomalous component behave as is expected from theory, with strong effects of gradient and curvature drifts. There is strong evidence of considerable latitude transport of the cosmic rays, at all energies, but the mechanism by which this occurs is unclear. Despite the apparent success of the theory, there is no single choice for the parameters which describe cosmic ray behavior, which can account for all of the observed temporal and spatial variations, spectra, and electron vs. ion behavior.
\end{abstract}

Space Science Reviews 83: 179-214, 1998.

(C) 1998 Kluwer Academic Publishers. Printed in Belgium. 


\section{Introduction}

The modulation of the intensity of galactic cosmic rays by the solar wind has been a subject of considerable interest for decades, for one primary reason: to understand the behavior of the cosmic rays with time over the solar cycle is to understand the behavior of the heliosphere as a whole. The heliosphere is vast. The supersonic solar wind extends to 80-100 AU from the Sun; subsonic solar wind flows a comparable distance beyond the solar wind termination shock (see Lazarus et al., 1998). Spacecraft observe only limited regions, at particular times. The highly mobile cosmic rays, however, sample all of the heliosphere, and their temporal and spatial evolution is a direct, but at times confusing response, to changing heliospheric conditions.

Current understanding of cosmic ray behavior in the heliosphere can be traced to the pioneering work of Parker (1965) and Gleeson and Axford (1967) who derived the fundamental equation which should govern the cosmic ray behavior:

$$
\frac{\partial n}{\partial t}=\nabla \cdot \kappa \cdot \nabla n-\nabla \cdot(\mathbf{V} n)-\mathbf{v}_{\mathbf{D}} \cdot \nabla n+\frac{1}{3} \nabla \cdot \mathbf{V} \frac{\partial}{\partial T}(\alpha T n)
$$

Here, $n$ is the differential number density of the cosmic rays, per unit kinetic energy $T$.

Each of the terms in this equation describes a separate physical process:

- The term on the left side is the variation of $n$ with time $t$. For many modulation applications, the cosmic rays are effectively in a steady state and this term can be neglected.

- The first term on the right describes the diffusion of the particles among the irregularities in the magnetic field of the solar wind. The diffusion tensor $\kappa$ has different components parallel and perpendicular to the magnetic field.

- The second term on the right describes the convection of the particles with the solar wind, with speed V.

- The third term is the drift of the particles in the mean magnetic field, expressed here in the useful formalism of an effective drift speed $\mathbf{v}_{\mathbf{D}}$, which, if the drift is unaffected by scattering or other mitigating effects, will be given by:

$$
\mathbf{v}_{\mathbf{D}}=\frac{c v p}{3 q} \nabla \times \frac{\mathbf{B}}{\mathbf{B}^{\mathbf{2}}}
$$

where $\mathbf{B}$ is the mean field strength, $p$ is particle momentum, $q$ is charge, and $c$ is the speed of light.

- The fourth term describes the adiabatic deceleration in the expanding solar wind, which was the fundamental contribution of Parker (1965). Here, $\alpha(T)=$ $\left(T+2 T_{0}\right) /\left(T+T_{0}\right)$, with $T_{0}$, the particle rest mass energy. It is also possible to express Equation (1) in terms of the distribution function of the cosmic rays in momentum space, which simplifies the adiabatic deceleration term (e.g. Fisk et al., 1973).

Equation (1) is a complicated, second order equation and needs to be solved numerically. Over the last several decades, sophisticated numerical codes have been 
developed at the University of Arizona and in South Africa, and are summarized in earlier chapters (Kóta and Jokipii; Potgieter; Jokipii and Giacalone, 1998). The tools exist, then, for detailed comparison of theory and observation. Certain insights from these analyses, and in some cases simply by inspection of Equation (1), are straightforward:

First, the cosmic ray behavior is sensitive to the configuration of the mean magnetic field, and the ability of particles to move perpendicular to the field. The diffusion coefficient parallel to the mean magnetic field is expected to be substantially larger than that perpendicular to the field, and thus the ready access of the cosmic rays will be determined by both the orientation of the field, and where the orientation is not favorable, by the perpendicular transport of the particles (see Giacalone, 1998). Similarly, the drift velocity given in Equation (2) depends on the field orientation, and perhaps on the scattering. As was pointed out in pioneering work of Jokipii et al. (1977), in a standard Archimedes spiral pattern for the solar wind magnetic field the drift speed can be several times the solar wind speed, and thus important in Equation (1). The drift velocity, however, may be modified by particle scattering or by other variations in the magnetic field (see Potgieter, 1998).

Second, the drift velocity is in opposite directions for electrons and ions, and also reverses with the polarity of the magnetic field, which results in overall flow patterns of cosmic rays in the heliosphere that vary substantially with charge and with the solar magnetic cycle (see Evenson, 1998). During the current solar minimum (1996; $q A>0$ ), ions should enter primarily over the solar poles, whereas electrons should enter along the equatorial current sheet; the opposite should be true in the previous solar minimum $(1987 ; q A<0)$. Similarly, the entry of ions over the solar poles, and their subsequent transport to lower latitudes should result in relatively small radial gradients, whereas, in the previous cycle, the entry of ions along the equatorial current sheet should result in larger radial gradients (see Potgieter, 1998). A comparison, then, between successive solar minima, and between the behavior of electrons and ions provide important tests of the role of particle drifts in modulation theory.

Third, the adiabatic deceleration is of fundamental importance in Equation (1). Its effects are of comparable magnitude to those of convection, which is the fundamental cause of the reduction of the cosmic ray intensity relative to that in the interstellar medium. Hence, we can expect that adiabatic energy loss will be negligible only when there is limited modulation, such as is now being sought by the Voyager mission in the outer heliosphere (see McDonald; Webber and Lockwood; Lazarus et al., 1998). Limited modulation was also a possibility for the region over the solar poles explored by Ulysses, but not seen (see McKibben, 1998). Thus, in all spectra seen in the inner heliosphere there is evidence for extensive energy loss by the particles. Equivalently, as was discussed in Goldstein et al. (1970) and Gleeson and Urch (1971),there is no information in these spectra about particles of low energy $(<100 \mathrm{MeV})$ in the interstellar medium. 
Fourth, Equation (1) works equally well for galactic cosmic rays and for the anomalous cosmic ray component which arises from interstellar neutral gas which is ionized in the solar wind and which is believed to be accelerated at the termination shock of the solar wind. Even the acceleration of the anomalous component can be included in Equation (1) since shock acceleration of mobile, energetic particles can be treated as a compression at the shock front, and its effects included in the fourth term on the right of Equation (1) (see Jokipii and Giacalone; Cummings and Stone, 1998). The behavior of galactic cosmic rays and the anomalous component thus provide related, but independent tests of modulation theory.

The comparison between the theory of cosmic ray behavior and the observations is most readily performed near solar minimum. Here, the heliosphere is wellordered into regions of high speed solar wind flow above and below the equatorial plane, emanating from the relatively steady polar coronal holes (see Suess et al., 1998). The field polarity is also well-ordered, with one polarity in the north, the opposite in the south, and a wavy current sheet in between, embedded in the region of slower solar wind flow near the equatorial plane (see Balogh, 1998).

The study of cosmic ray behavior near solar minimum thus is essentially an effort to answer two fundamental questions: First, is the basic theory correct? Does Equation (1), as expected, capture all of the major physical effects? And second, if it does, what does the cosmic ray behavior tell us about the overall structure and evolution of the heliosphere?

In this chapter we explore these two questions with a series of short sections each of which discusses a different aspect of the global behavior of cosmic rays near solar minimum.

- We begin by describing the evolution of the intensities and spatial gradients of the cosmic rays, in successive solar minima. Such evolution reveals the overall flow patterns of the cosmic rays, as they recover to their maximum intensity at solar minima.

- We discuss the latitude gradients of the cosmic rays, particularly as seen from Ulysses, and also the curious feature observed by Ulysses that there is an apparent offset in the plane of symmetry of the modulation. The latitude gradients are small and our understanding of them needs to be consistent with the overall flow patterns of the cosmic rays.

- We discuss the anomalous component and how it evolves between successive solar minima. The anomalous component, which has steep spectra, can be more sensitive to the effects of adiabatic deceleration, and thus provides important tests of our understandings; in contrast, the acceleration of the anomalous component should vary in time which may confuse the interpretation of its behavior.

- We consider the differences between the behavior of cosmic ray electrons and ions, which places important constraints on the role of particle drifts in modulation. 
- We consider 26-day variations in the cosmic rays, which are seen up to the highest latitudes observed by Ulysses. This unexpected observation places important constraints on the ability of and the mechanism by which particles are transported in latitude (see also Simpson, 1998).

The conclusions of these sections and their implications for our assessment of the global processes which control cosmic ray modulation are summarized in the final section.

\title{
2. Study of the Radial Intensity Gradients of Galactic Cosmic Rays
}

\author{
F. B. McDonald and Z. Fujii
}

\subsection{INTRODUCTION}

The radial intensity gradient of cosmic rays in the heliosphere is a valuable means of studying the temporal and spatial variations in the extensive data now available from the Pioneer, Voyager, Ulysses and IMP missions. Many previous studies have been primarily concerned with radial gradients of integral counting rates (Venkatesan $e t$ al., 1984; Lockwood, 1985; McKibben, 1987; Lopate and Simpson, 1991; Fillius, 1989). It has generally been difficult to use these data to delineate spatial from temporal change. In this contribution we focus upon the radial gradient for galactic cosmic ray $\mathrm{He}(180-450 \mathrm{MeV} / \mathrm{n})$ and $\mathrm{H}(130-220 \mathrm{MeV})$ near the ecliptic plane over the 1972.25-1996.0 time period.

The local intensity gradient at a given location, $g_{r}$, is defined by the relation

$$
\frac{1}{J} \frac{d J}{d r}=g_{r}
$$

Actual measurements are generally between two widely separated spacecraft which give what is termed the non-local gradients $G_{r}$ (Potgieter et al., 1989)

$$
G_{r}=\frac{1}{\left(r_{2}-r_{1}\right)} \ln \frac{J_{2}}{J_{1}}
$$

However, the data over successive solar minima (Webber and Lockwood, 1985; Cummings and Stone, 1988; Cummings et al., 1990; McDonald et al., 1992) indicate that these radial gradients are a function of heliocentric distance with a spatial dependence that is consistent with:

$$
\frac{1}{J} \frac{d J}{d r}=G_{o} r^{\alpha}
$$

and for measurements at two spacecraft:

$$
\ln \frac{J_{2}}{J_{1}}=G_{o} \frac{r_{2}^{\alpha+1}-r_{1}^{\alpha+1}}{\alpha+1} \text {. }
$$



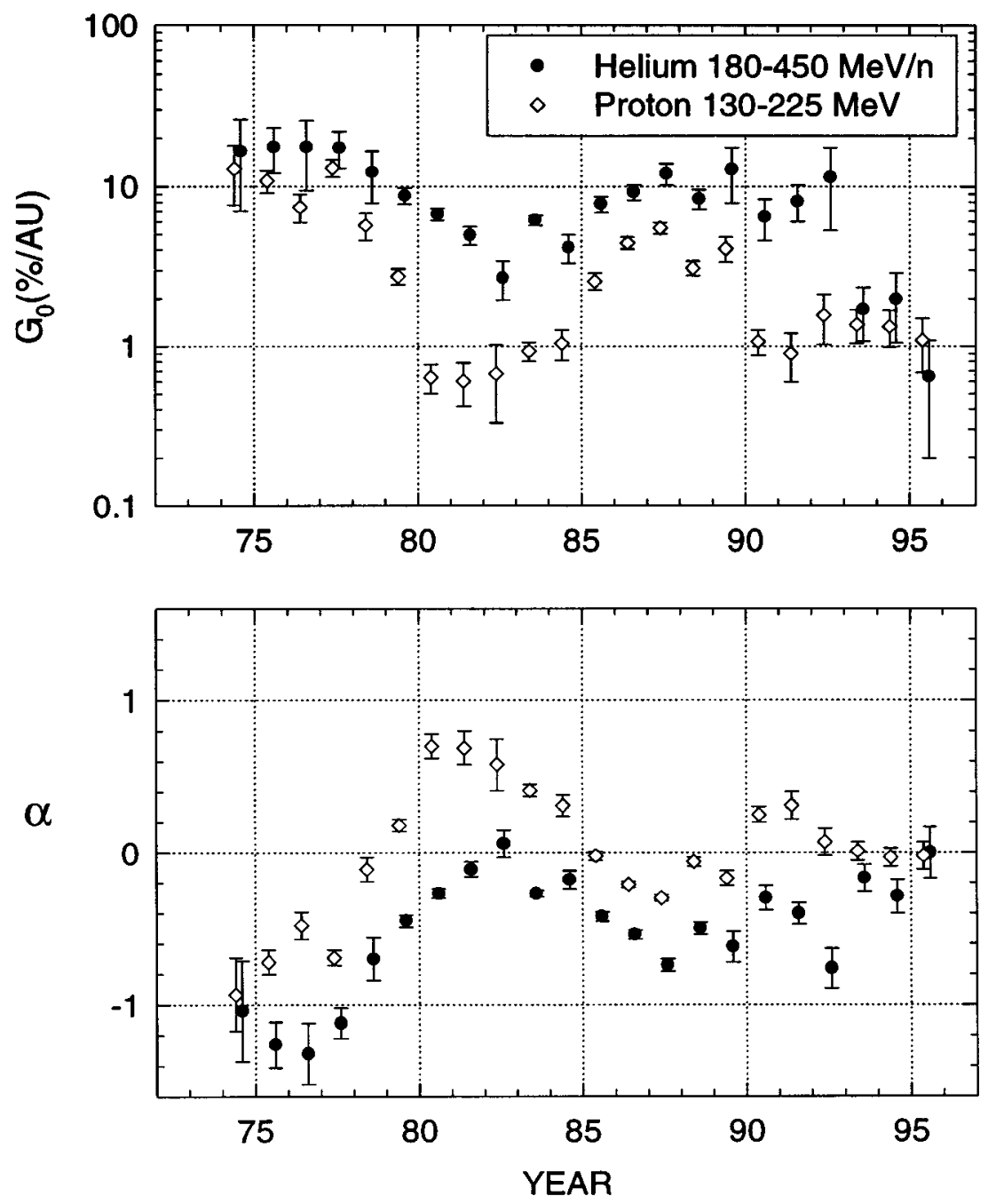

Figure 1. Values of $G_{o}$ and $\alpha$ (12-month averages) obtained from the relation $g_{r}=G_{o} r^{\alpha}$ for $180-450 \mathrm{MeV} / \mathrm{n} \mathrm{He}$ and 130-220 MeV H (Fujii and McDonald, 1997).

A minimum of three spacecraft at different radial distances is required for the determination of both $G_{o}$ and $\alpha$. Fortunately, such data are available over the 1973-1995 time period. To the extent that this is a valid representation of the radial dependence of the radial gradient, then $g_{r}=G_{o} r^{\alpha}$.

The annual values of $G_{o}$ and $\alpha$ (Figure 1) for galactic cosmic-ray $\mathrm{H}$ and $\mathrm{He}$ display reasonably consistent changes over most of solar cycle 21 and 22 and an $\sim 11$-year cycle pattern in their variation. For the 1980 and 1990 solar maxima the values of $G_{o}$ and $\alpha$ are more nearly the same, but there is a significant change in $\alpha$ 
that is believed to be due to drift effects. For both cycles there are large changes in the radial dependence of $\alpha$ between solar minimum and solar maximum.

\subsection{DISCUSSION}

With the relation $g_{r}=\frac{1}{J} \frac{d J}{d r}=G_{o} r^{\alpha}$ and the sets of values of $G_{o}$ and $\alpha$ it becomes possible to estimate the values of $g_{r}$ for galactic cosmic-ray $\mathrm{H}$ and $\mathrm{He}$ near the plane of the ecliptic out to the limits of the modulation boundary. This extrapolation can be done either as a function of radial distances (Figure 2) at a given time, or as a function of time for a fixed radial distance (Figure 3).

The estimates of the $\mathrm{H}$ and $\mathrm{He}$ gradients from 1-100 AU for the 1977 and 1987 solar minimum periods as well as the 1981 and 1990 solar maxima are shown in Figure 2. At the time of the two successive solar minima, the $\mathrm{H}$ and $\mathrm{He}$ intensity gradients become very small with increasing heliocentric distance. However, for both galactic cosmic-ray $\mathrm{H}$ and $\mathrm{He}$ at all radial distances the 1987 values of $g_{r}$ are larger than in 1977. For He these values differ by a factor of $\sim 3$ over the 40-60 AU interval. These smaller values of $g_{r}$ in 1977 occur in a $q A>0$ epoch (1970-1980) when cosmic-ray ions are drifting in over the solar poles and leave via the heliospheric neutral current sheet and are in broad agreement with the prediction of drift theory (Jokipii and Thomas, 1981; Kóta and Jokipii, 1983; Potgieter et al., 1989).

At solar maximum the values of $G_{o} r^{\alpha}$ (Figure 2) are appreciably higher than at solar minimum. Furthermore, the $\mathrm{H}$ gradients are increasing with increasing heliocentric distance. Such a radial dependence was also obtained by Potgieter et al. (1989) using both 1-dimensional and 2-dimensional non-drift modulation models and diffusion coefficients suitable for these time periods.

The annual values of $g_{r}$ for $\mathrm{H}$ and He over the 1974.0-1996.0 period for 1, 10, and $75 \mathrm{AU}$ (Figure 3) illustrate the complex changes that occur in $g_{r}$ for both components over the solar and heliomagnetic cycle. For He the $1 \mathrm{AU}$ gradients decrease going from 1977 to 1982 while the 10 and 75 AU gradients increase in such a manner that the three data sets converge near a common value of $3 \% / \mathrm{AU}$ in 1982. The behavior of lower rigidity $\mathrm{H}$ over this time period is marked by a crossover in 1979. There are no significant changes in $g_{r}$ for $\mathrm{H}$ or He associated with the reversal of the solar magnetic field in 1980 and 1990.

Starting about 1993.0 the cyclic patterns in $G_{o}, \alpha$ and $g_{r}$ are no longer present. This change may be a result of the increasingly larger heliocentric distance of Voyager 2 and Pioneer 10, the absence of any measurements at intermediate distances, and the possibility that the radial dependence of $g_{r}$ may be more complex than that used in the present analysis. For example, it is often assumed that the particle diffusion coefficient is inversely proportional to the magnitude of the interplanetary magnetic field. However, in the inner heliosphere the interplanetary magnetic field in the ecliptic plane decreases as $\sim 1 / r^{2}$ and as $\sim 1 / r$ in the outer heliosphere. 

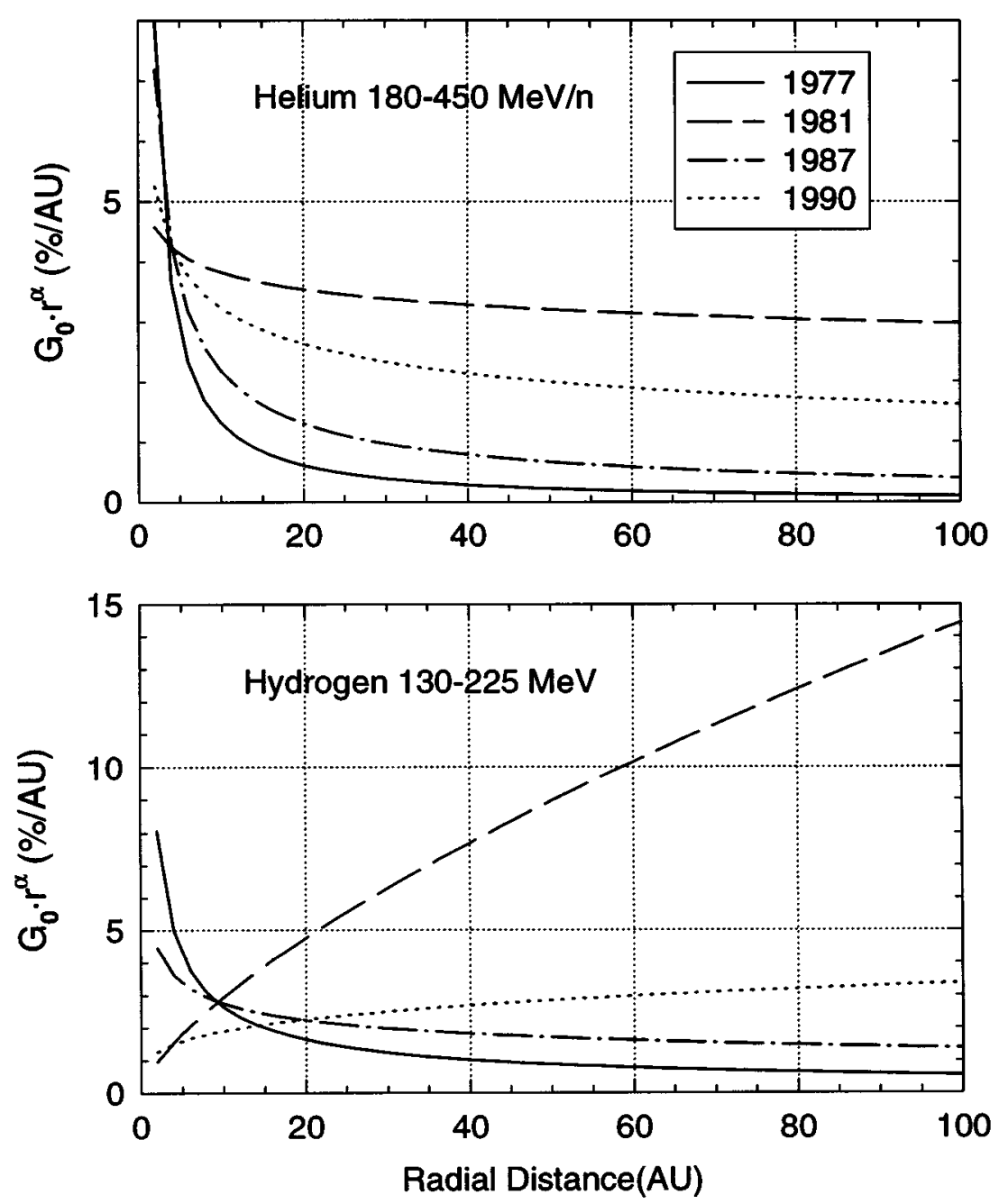

Figure 2. The estimated values of $g_{r}$ as a function of heliocentric distance at the times of successive solar minima and maxima using the relation $g_{r}=G_{o} r^{\alpha}$ and the appropriate values of $G_{o}$ and $\alpha$ shown in Figure 1 (from Fujii and McDonald, 1997).

In previous studies of the radial dependence of $g_{r}$, Cummings et al. (1995) obtained a value of $\alpha=-1.7$ for $10 \mathrm{MeV} / \mathrm{n}$ anomalous oxygen $(R=2.2 \mathrm{GV})$ over the last half of 1993 using data from Ulysses, Sampex at 1 AU and Pioneer 10 and the Voyagers in the outer heliosphere. This compares with $\alpha=-0.96$ for the first half of 1987 (Cummings et al., 1990). McDonald et al. (1992) found that in 1987 anomalous $\mathrm{He}$ and $175-450 \mathrm{MeV} / \mathrm{n} \mathrm{He}$ had the same radial dependence of $\alpha=-0.7$. Except for 1993, these values are in reasonable agreement with the results presented in this paper for the comparable time periods. McDonald et al. (1997) found values of $\alpha=-0.66 \pm 0.08$ and $-0.82 \pm 0.07$ for $145-255 \mathrm{MeV} / \mathrm{n}$ 

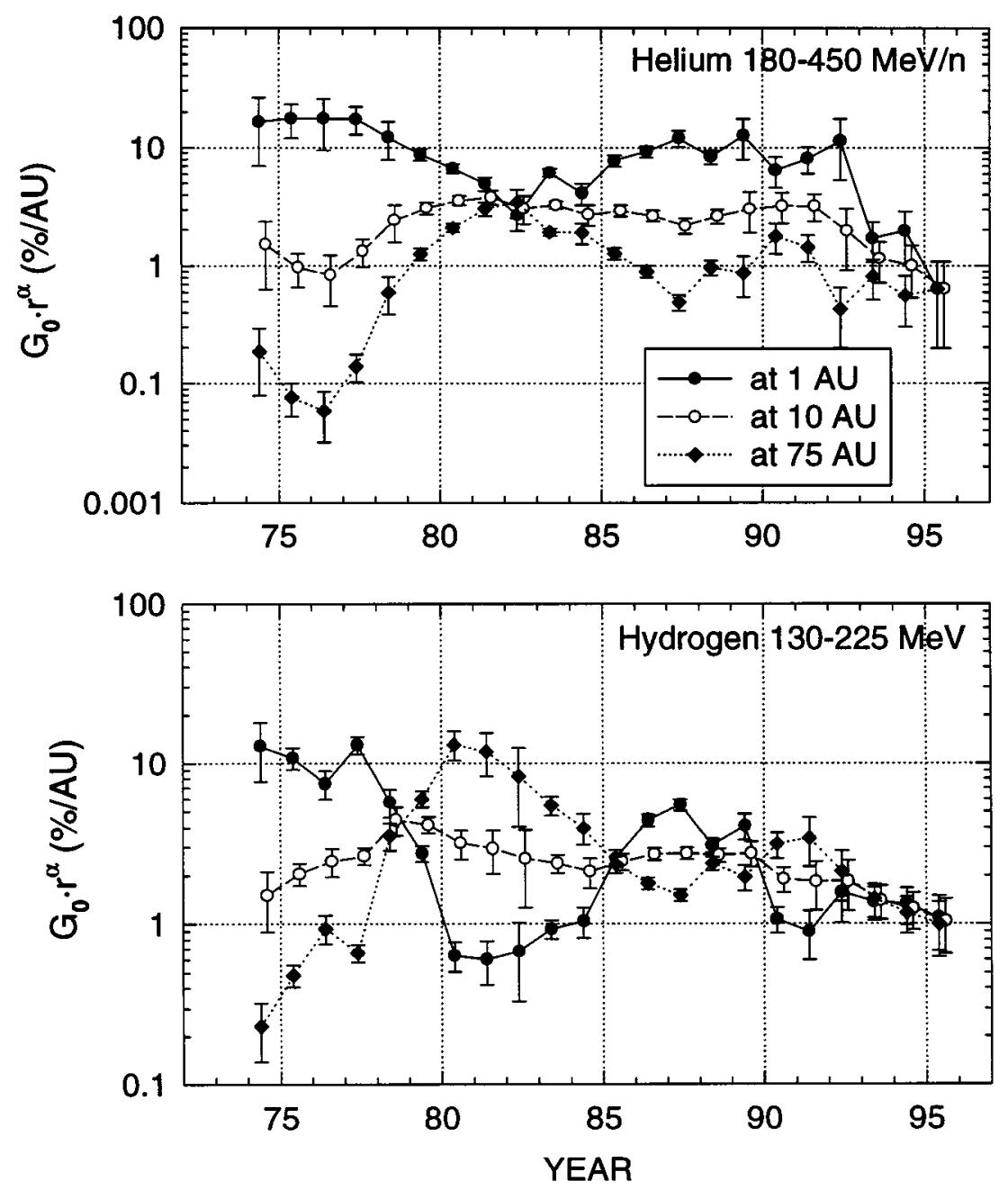

Figure 3. The estimated values of $g_{r}$ for galactic cosmic-ray He and $\mathrm{H}$ over the 1974.0-1996.0 time period for 1,10 and $75 \mathrm{AU}$ using the relation $g_{r}=G_{o} r^{\alpha}$ and the values of $G_{o}$ and $\alpha$ (from Fujii and McDonald, 1997).

He for time periods in early 1993 and 1994 using data from Ulysses, Voyager 2, Pioneer 10 and IMP 8. These 1993/1994 results using Ulysses at intermediate distance (4-5 AU) suggest that the major difficulty with the present analysis may be the increasingly large radial distance of the three spacecraft in the outer heliosphere and a small departure from the $G_{o} r^{\alpha}$ representation.

Since 1977 and 1995 are close to the same phase of both the heliomagnetic and solar cycle, it is possible to predict the expected gradient at $55 \mathrm{AU}$ and compare it with that measured in 1996. This 1977 data predicts a gradient for $315 \mathrm{MeV} / \mathrm{n} \mathrm{He}$ at $55 \mathrm{AU}$ of $g_{r}$ of $0.2 \pm 0.09 \% / \mathrm{AU}$ while the measured 1995 value is $0.33 \pm 0.14$ 
using the Pioneer 10, Voyager 1 and Voyager 2 data. For $\mathrm{H}$ the measured value is $0.98 \pm 0.1 \% / \mathrm{AU}$ versus a predicted value of $0.7 \pm 0.15$ using the 1977 parameters.

The next step in these studies is to use the Ulysses cosmic-ray data at intermediate radial distances to study $g_{r}$ in the 1996-1997 period when that spacecraft is near the ecliptic plane.

\title{
3. Latitudinal Structure of Modulation in the Inner Heliosphere
}

\author{
R. B. McKibben, R. A. Burger, B. Heber, J. R. Jokipii, F. B. McDonald and
}

M. S. Potgieter

\subsection{INTRODUCTION}

Since the cosmic ray source is constant over the outer boundary of the heliosphere, nonuniformities of the intensity within the heliosphere provide direct information about spatial variations in the effectiveness and relative importance of the physical processes that produce the modulation. Thus the study of spatial gradients of the cosmic ray intensity plays a very significant role in understanding the transport of cosmic rays through the heliosphere.

Prior to Ulysses' exploration of the high latitude regions of the inner heliosphere, essentially all of our information about spatial gradients was limited to a narrow range within about $30^{\circ}$ of the ecliptic plane. Thus, while the radial evolution of the modulation was well characterized by detailed studies out to beyond $60 \mathrm{AU}$ (McDonald et al., 1997), very limited information was available concerning the latitudinal structure of modulation. While this remains the case in the outer heliosphere, Ulysses has provided a rather complete picture of the latitudinal variations in modulation of galactic cosmic rays, anomalous components, and electrons between the latitudes of $80^{\circ} \mathrm{S}$ and $80^{\circ} \mathrm{N}$ at radii between 1 and $5 \mathrm{AU}$. Since the observations and their interpretation are discussed in more detail in other chapters of this publication, only the primary conclusions are summarized here.

\subsection{The UlysSES OBSERVATIONS}

The most reliable observations of latitude effects are those performed during the fast latitude scan consisting of the approximately 1 year period centered on Ulysses perihelion during which the spacecraft traversed the latitude range from $80^{\circ} \mathrm{S}$ to $80^{\circ} \mathrm{N}$ while staying within the radial range 1.3-2.3 AU. The level of modulation as measured by IMP 8 and Earth-based detectors was also relatively stable during this period, so that there was relatively little contribution to intensity changes at Ulysses during the fast latitude scan from either temporal changes in modulation or from effects of radial gradients. While radial gradients for some species have been reported from Ulysses measurements (Heber et al., 1997b), not all of the reports of 
latitude variations have made corrections for the effect of radial gradients. However, because of the small radial range during the fast latitude scan and the small size of radial gradients (a few percent per AU), incorporation of such corrections would in general make only small changes in the values of the reported latitude gradients.

The latitude gradients found by Ulysses for galactic cosmic ray nuclei and anomalous components were positive, with intensities increasing towards the poles. This is consistent with predictions of modulation models that include the effects of gradient and curvature drifts for the current positive polarity of the Sun's dipole magnetic field $(q A>0)$. The measured gradients are summarized as a function of particle magnetic rigidity in Figure 4, including both the inner heliosphere gradients measured by Ulysses and gradients measured in 1996 in the outer heliosphere by the Pioneer 10 and Voyager 1 and 2 spacecraft over the latitude range from $19^{\circ} \mathrm{S}$ to $34^{\circ} \mathrm{N}$. Latitude gradients measured in the outer heliosphere appear to be comparable to or somewhat smaller than those measured by Ulysses. All measured gradients are small, at most $\sim 2 \% /$ degree for certain of the anomalous components, and less than $1 \% /$ degree for galactic cosmic rays. The anomalous component gradients are comparable to those measured in the previous solar minimum with positive magnetic polarity (McKibben, 1989) and significantly smaller than the negative gradients measured within $30^{\circ}$ of the ecliptic in the 1987 solar minimum with negative solar magnetic polarity (Cummings et al., 1987). From Figure 4, the largest latitudinal gradients are observed for particles with rigidities near $1 \mathrm{GV}$. For galactic cosmic rays the measured values decline steeply from the maximum to near-zero for rigidities of the order of a few hundred MV, and decline more gradually towards high rigidities. Consistent with the small gradients, the modulated spectra observed at the poles are very similar to those observed in the ecliptic for both galactic cosmic rays and anomalous components.

For the flux of electrons $>300 \mathrm{MeV}$, contrary to expectations based on simple implementations of drift-dominated modulation models, no obvious latitude dependence was observed (Ferrando et al., 1996). Accounting for this lack of latitude dependence has become one of the challenges posed to comprehensive 3-dimensional modulation models by the Ulysses observations.

While the gradients for nucleons are small and positive, they are not uniform from the equator to the polar regions. Shortly after Jupiter flyby, Ulysses spent an extended period near $5 \mathrm{AU}$ and at latitudes less than $30^{\circ}$ in the region swept by the heliospheric current sheet. During this period, little variation in the intensities of cosmic ray nuclei and anomalous components with latitude was observed. During the fast latitude scan, passage through the region swept by the current sheet was so rapid that the suppression of latitudinal effects in the current sheet region near the perihelion radius of $1.3 \mathrm{AU}$ could not be assessed conclusively. Recent observations during Ulysses' return to low latitudes near the orbit of Jupiter appear to confirm the effect of the current sheet in suppressing latitude gradients near 5 AU (Heber et al., 1997b). 

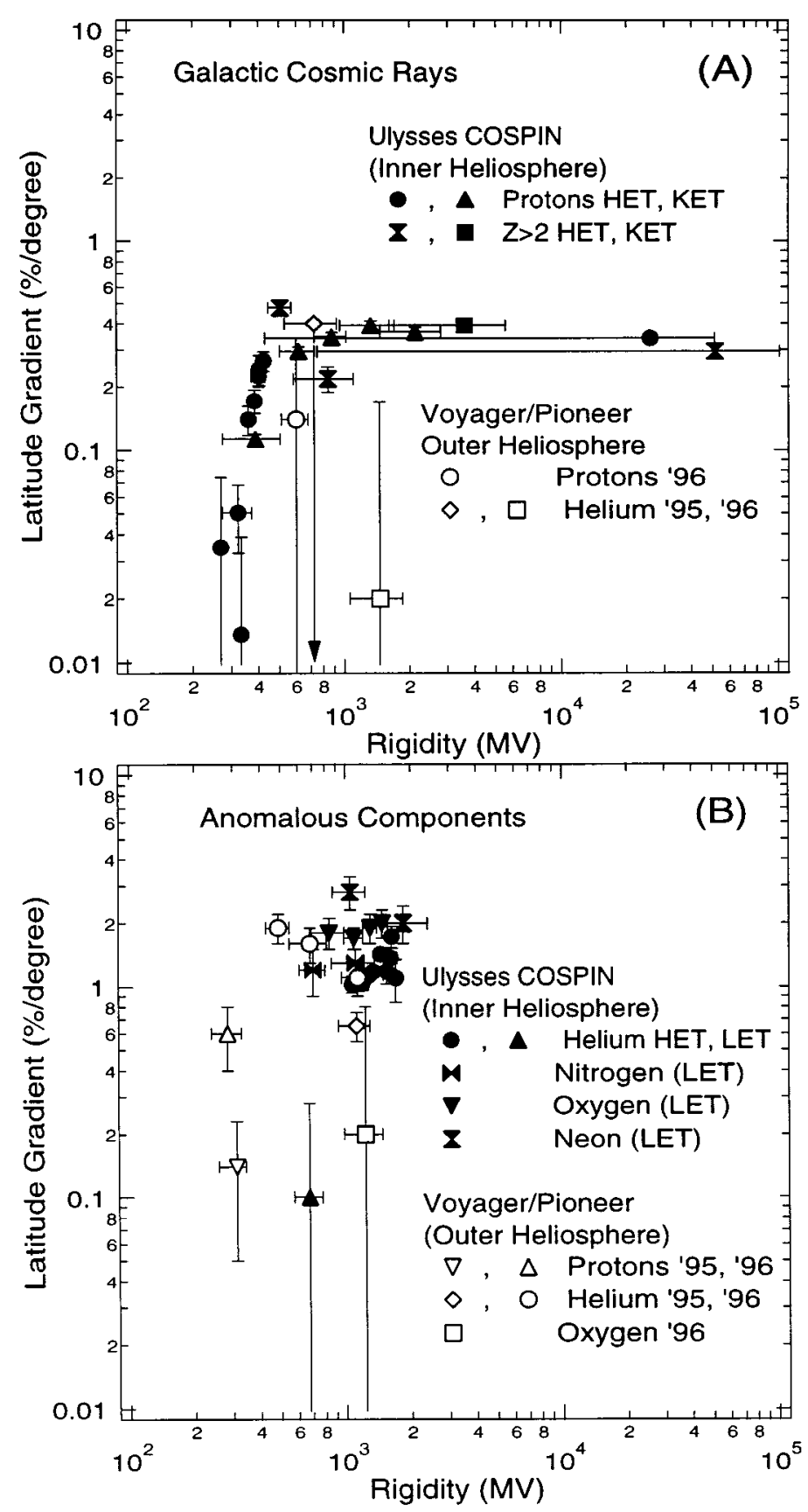

Figure 4. Measured latitudinal gradients 1994-1996 as a function of particle magnetic rigidity for galactic cosmic rays (A) and anomalous components (B). Ulysses measurements are from Trattner et al. (1995, 1996), Heber et al. (1996a), and McKibben et al. (1996). Most Pioneer/Voyager measurements are from McDonald et al. (1997). 
Above the regions swept by the current sheet, the intensities of particles with rigidities $>500 \mathrm{MV}$ increased steadily (when compared to simultaneously measured intensities in the ecliptic) up to latitudes of about $60^{\circ} \mathrm{S}$, above which little variation with latitude was observed (Simpson et al., 1995). During the fast latitude scan the near absence of a latitude gradient at the highest latitudes was confirmed for both the north and the south polar regions (McKibben et al., 1996; Heber et al., 1996a). However more recent observations reported by Heber et al. (1997b) for the period after the north polar pass do not show this effect as clearly.

A very surprising observation made during the fast latitude scan, first remarked upon by Simpson (1996), was that the plane of symmetry of the modulation appeared to be offset southwards from the heliographic equator by about $10^{\circ}$, and that the fluxes observed over the north polar regions, when compared to simultaneous in-ecliptic measurements, were about $10 \%$ larger than the fluxes over the south polar regions, implying a strong north-south asymmetry to the modulation in the heliosphere. Figure 5, clearly shows the southward offset of the minimum intensity for the integral intensity of protons $\gtrsim 100 \mathrm{MeV}$ (Figure 5A). Furthermore, as shown in Figure $5 \mathrm{~B}$ when the intensities are reflected about a latitude of $10^{\circ} \mathrm{S}$, a near-perfect alignment of intensities in the northern and southern hemispheres is obtained (i.e. the latitudinal gradients measured with respect to $10^{\circ} \mathrm{S}$ are the same in both hemispheres), suggesting a possible interpretation of the north polar flux excess as simply the result of the larger latitudinal range available for the gradient to act through in the northern hemisphere. Similar behavior was observed for anomalous helium (Simpson et al., 1996; McKibben et al., 1996) and for $>2 \mathrm{GeV}$ protons (Heber et al., 1997a).

No gross features of either the interplanetary magnetic field or the solar wind velocity structure measured by Ulysses have been found to correlate with this southward offset. The observed constancy of the radial magnetic field components as a function of latitude (Smith and Balogh, 1995; Suess et al., 1996), together with the requirement that $\operatorname{div}(\mathbf{B})=0$, implies that the heliospheric current sheet separating the north and south magnetic hemispheres cannot on average be suppressed $10^{\circ}$ south of the equator. In the solar wind, the sharp increases the solar wind speed defining the boundaries of the equatorial solar wind were observed to lie symmetrically approximately $20^{\circ}$ north and south of the equator (Phillips et al., 1995). As a result, the reality of the southward offset of modulation has been the subject of much discussion at this Workshop.

For the southward displacement of the minimum flux, it was suggested that as a result of the speed with which Ulysses passed through the equatorial zone, the relative positions of Ulysses and of IMP-8 (to which the Ulysses fluxes were being compared) with respect to the corotating undulations in the heliospheric equatorial current sheet at the time of Ulysses' equator crossing could account for the apparent offset. Analysis of the effect of artificially advancing or delaying the Ulysses data with respect to the IMP data by periods of up to half a solar rotation showed that the apparent offset of the absolute minimum Ulysses/IMP flux ratio from the equator 


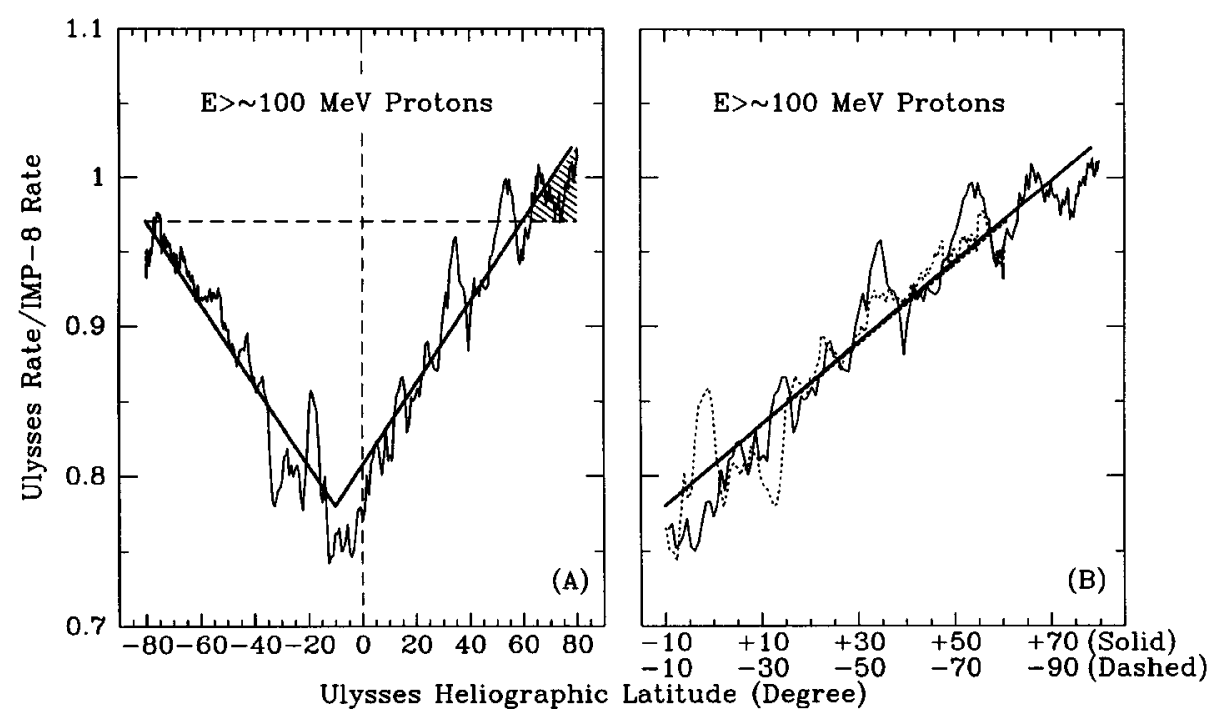

Figure 5. A) Daily average Ulysses/IMP counting rate ratios as a function of heliographic latitude for the integral intensity of protons $>100 \mathrm{MeV}$. B) The same observations reflected about a latitude of $10^{\circ} \mathrm{S}$ and superposed to show the symmetry of the modulation about this latitude. Lines are least squares fits to the data (from Simpson et al., 1996).

could indeed vary by as much as $10^{\circ}$ in latitude, However, knowledge of the actual current sheet structure and of the spacecraft positions with respect to it at the time, which have not yet been folded into this analysis, does not allow free choice of the relative phasing of the Ulysses and IMP observations. Furthermore, whatever the case for the minimum, another explanation is required for the organization of the high latitude observations with respect to $10^{\circ} \mathrm{S}$ latitude since at the higher latitudes Ulysses latitudinal velocity has slowed to the point that significant motion in latitude takes place only over periods comparable to a solar rotation. As a result, the intensities in any given latitudinal band represent an average over solar rotation, and phasing with respect to the current sheet undulations is not likely to explain the apparent symmetry with respect to $10^{\circ} \mathrm{S}$.

\subsection{THEORETICAL IMPLICATIONS OF THE OBSERVATIONS}

The Ulysses observations of the latitudinal structure of modulation are still in the process of being assimilated into models of the global heliospheric modulation. However some of the implications are already clear.

The small size of the latitudinal gradients observed clearly implies that access of cosmic rays to the inner heliosphere is little easier over the poles than in the ecliptic plane, despite the looser winding of the ideal Parker model interplanetary magnetic field lines expected over the poles. This, together with direct observations of large amplitude Alfvén waves in the high latitude magnetic field (Forsyth et al., 
1996), appears to confirm the suggestion by Jokipii and Kóta (1989) that transverse fluctuations in high latitude magnetic field lines may play a major role in the controlling propagation of energetic particles through the polar heliosphere. With access thus impeded, the polar heliosphere is much like the equatorial heliosphere for low energy particles, and the modulated spectra are determined by the same balance between convection and adiabatic deceleration as in the ecliptic plane. As Paizis et al. (1997) have noted, this results in a Compton-Getting factor near zero for low energy galactic cosmic rays, consistent with the near-absence of spatial gradients for these particles.

To achieve even reasonably acceptable fits to the observations using current three-dimensional numerical modulation models, and to explain other features of the modulation such as the persistence of 26-day periodic variations in modulation to the highest latitudes (see report of Working Group 2), it has further proved necessary to enhance latitudinal transport of energetic particles in the heliosphere. Within the models this has so far been implemented by incorporating an anisotropy in diffusion perpendicular to the magnetic field (Potgieter, 1997; Potgieter et al., 1997), with a latitudinal diffusion coefficient increased to values as large as $30 \%$ of the value of the diffusion coefficient parallel to the field. As an alternative, with however the same goal of enhancing latitudinal transport, Fisk (1996) has suggested a new model for the geometry of the interplanetary field. In his model, the interaction of the differential rotation of the photosphere with the non-differentially rotating overlying solar magnetic structure leads to establishment of direct magnetic connections between the polar and equatorial regions of the heliosphere.

The effect of enhancing latitudinal transport is to enhance the importance of diffusion and to diminish the effectiveness of gradient and curvature drifts in controlling the modulated intensities in the inner heliosphere at all latitudes. Potgieter et al. (1997) find that with such modifications to their standard model, they are able to reasonably reproduce both the small gradients for nucleons and the near-absence of latitude effects for highly relativistic electrons in the inner heliosphere. With further modifications, for example by incorporating the tighter winding for the south polar interplanetary magnetic field found by Forsyth et al. (1995), Hattingh et al. (1997) have also reproduced the southward offset of the symmetry of the modulation and the north polar flux excess.

Nevertheless, the reconciliation of Ulysses observations of the latitudinal structure of modulation with modulation models is still in a very preliminary state. Much more exploration of the range and functional forms of the parameters of the models, and perhaps even fundamental rethinking of some of our understanding of the transport of energetic particles through the heliosphere will be required before full understanding is achieved. 


\section{Anomalous Cosmic Rays and the 22 Year Solar Modulation Cycle}

W. R. Webber, J. R. Jokipii, J. Kóta, J. A. Lockwood, C. D. Steenberg, H. Moraal, M. S. Potgieter, J. A. Le Roux, F. B. McDonald, A. C. Cummings and E. C. Stone

\subsection{INTRODUCTION}

As the current solar 11-year cycle reached its minimum in 1996, it has become clearly evident that the intensities of anomalous $\mathrm{H}, \mathrm{He}$, and $\mathrm{O}$ nuclei are greatly different than they were at the minimum of the previous cycle in 1987. In the outer heliosphere between 40-60 AU the intensities are typically only $0.1-0.2$ of what they were at the same radial distance in the previous cycle (Stone et al. 1996; Webber and Lockwood, 1997; McDonald and Lal, 1995). The situation at the Earth seems to be reversed with the intensities in 1996 actually larger than those observed in 1987 (Lockwood and Webber, 1997). Obviously the radial gradients must be quite different as well in the two cycles. This data provides clear evidence for a solar magnetic polarity effect on the anomalous component intensities. Such effects are the basis of models for the solar modulation which include drift effects; however, the large intensity differences that are observed between cycles require other explanations beyond simple drift effects within the region of the heliosphere inside the solar wind termination shock (SWTS).

\subsection{Observations AND Model CAlCulations}

In an attempt to understand the situation we have examined the intensities of 10$22 \mathrm{MeV} / \mathrm{n} \mathrm{He}^{+}, 7-17 \mathrm{MeV} / \mathrm{n} \mathrm{O}^{+}$and $30-60 \mathrm{MeV} \mathrm{H}^{+}$as a function of heliocentric radius, $R$, near the equatorial plane, comparing both measurements and predictions for the negative polarity cycle minimum in 1987 , and the positive polarity minimum 1996. These results are shown in Figures 6, 7, and 8. The calculations are shown for shock locations at 70 and $85 \mathrm{AU}$ for a nominal set of interplanetary diffusion parameters: $\kappa(R) \propto R$ and $\kappa(P) \propto P(P$ is the particle rigidity). The data come from the Pioneer 10 (P10), Voyager 2 (V2) and IMP 8 spacecraft at 1987.5 and 1996.0. At 1987.5, P10 and V2 are located at 42 and 24 AU respectively, and in 1996.0 these distances are 64 and 47 AU. The large intensity difference between cycles in the outer heliosphere is immediately apparent in the data for $\mathrm{He}^{+}$and $\mathrm{O}^{+}$and is much less obvious in the data for $\mathrm{H}^{+}$. A crossover of intensities near the Earth is seen for $\mathrm{He}^{+}$and $\mathrm{O}^{+}$; however, the intensity of $\mathrm{He}^{+}$is too small near Earth to make a clear observation of this effect.

The model calculations likewise show this large difference of a factor $\sim 5$ 10 between cycles for the $\mathrm{He}^{+}$and $\mathrm{O}^{+}$intensities in the outer heliosphere. The observed crossover of intensities between cycles just beyond the Earth is also correctly predicted. The predicted difference between $\mathrm{H}^{+}$intensities in the positive and negative cycles is much less than that for the other two species, in agreement with 


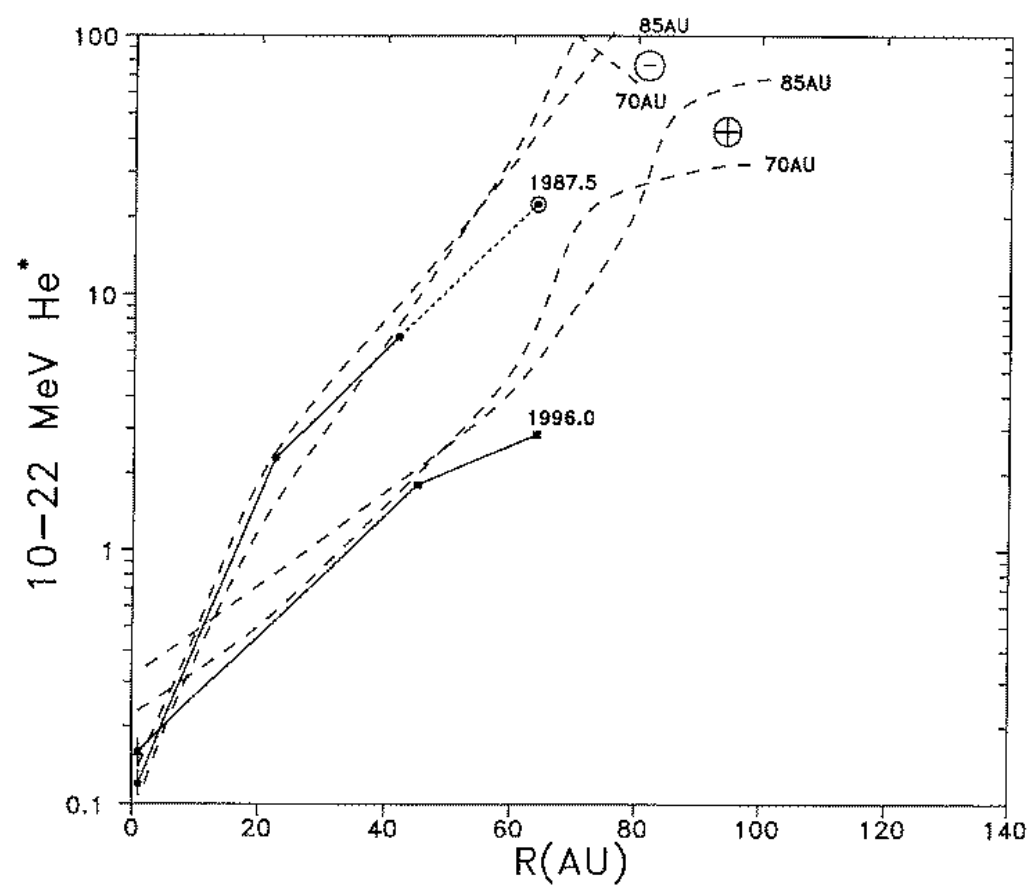

Figure 6. Differential intensities of $10-22 \mathrm{MeV} / \mathrm{n} \mathrm{He}^{+}$nuclei as a function of heliocentric radius. P10, V2, and IMP data points are shown as solid symbols connected by solid lines. Predictions for positive (+) and negative (-) cycles for two possible termination shock distances are shown as dashed lines.

the data. So overall the model calculations correctly predict the general behavior of the anomalous nuclei intensities in the opposite polarity cycles. They show that this difference is a result of a much lower intensity near the heliospheric equator at the location of the termination shock at the time of the positive cycle minimum.

Overall the radial dependence of the intensities in the two cycles fit slightly better with a termination shock location at $85 \mathrm{AU}$. This fit can be optimized by normalizing the intensities at the termination shock and varying the radial dependence of $\kappa(R)$. Further work is in progress in this area to try to determine the location of the shock more accurately. The rigidity dependence of $\kappa(P)$ will determine the relative changes in the various components in the two polarity cycles, as well as the extrapolation to the termination shock. The range in rigidity is from $\sim 2.4 \mathrm{GV}$ for $\mathrm{O}^{+}$to $\sim 0.3 \mathrm{GV}$ for $\mathrm{H}^{+}$. The total extrapolation to the termination shock is a factor $\sim 1.5$ for $\mathrm{O}^{+}$at $64 \mathrm{AU}$, a factor $\sim 10$ for $\mathrm{He}^{+}$, increasing to a factor $\sim 100$ for $\mathrm{H}^{+}$, in inverse order to their rigidity. Here the computational effort, which consists of varying $\kappa(P)$, is directed towards determining accurate intensities and spectra at the termination shock during both cycles.

The overall comparison in this section is, of course, restricted to near the equator (latitudes $<35^{\circ}$ ), because that is where the spacecraft are located. The 


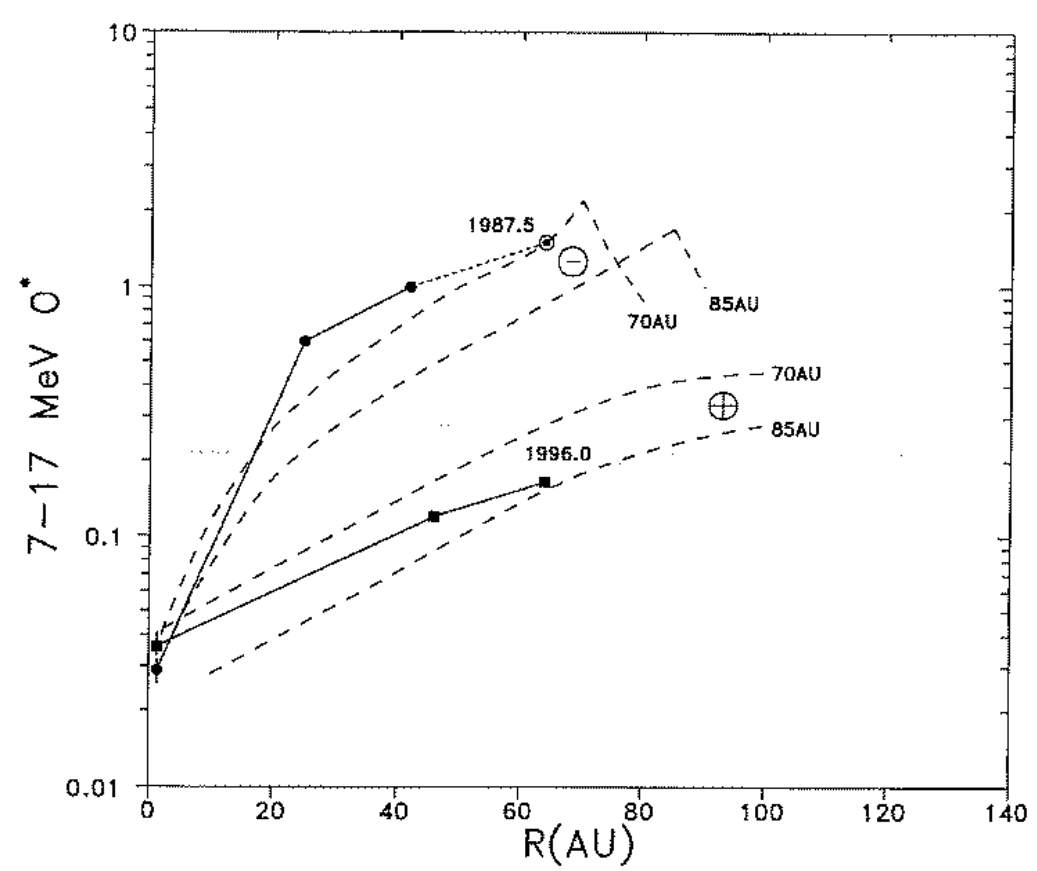

Figure 7. Same as Figure 6 but for $7-17 \mathrm{MeV} / \mathrm{n} \mathrm{O}^{+}$.

calculations show that in the negative cycle there is a negative intensity gradient along the termination shock leading to much smaller intensities at the pole (see e.g. Figure 9C). This result is at least partly responsible for the negative latitudinal gradients observed within the heliosphere at this time (1987) (see Stone et al., 1996). In the positive cycle the calculations show the opposite behavior, a positive gradient along the termination shock leading to much larger intensities at the pole and again responsible for the positive latitudinal gradients seen within the heliosphere at this time (1996).

We note that the high intensities at the shock near the equator in the negative cycle are roughly, but not exactly, matched by the high intensities at the shock near the pole in the positive cycle. This alternating intensity behavior near the shock in the two cycles is a result of the drift and acceleration of the anomalous cosmic rays along the shock (Jokipii, 1990), but the effective accelerating potential, $\phi$, is not quite symmetric between both cycles, being more effective in the negative cycle when the particles are drifting along the shock from the pole to the equator. The important thing to note here is, however, that the "source" function for anomalous cosmic rays is changing dramatically along the shock from negative to positive cycles. This in turn has a large effect on the anomalous intensities within the heliosphere. In fact a large part of the observed "modulation" of the anomalous components within the heliosphere at all locations may simply be the result of these intensity changes taking place near the solar wind termination shock. 


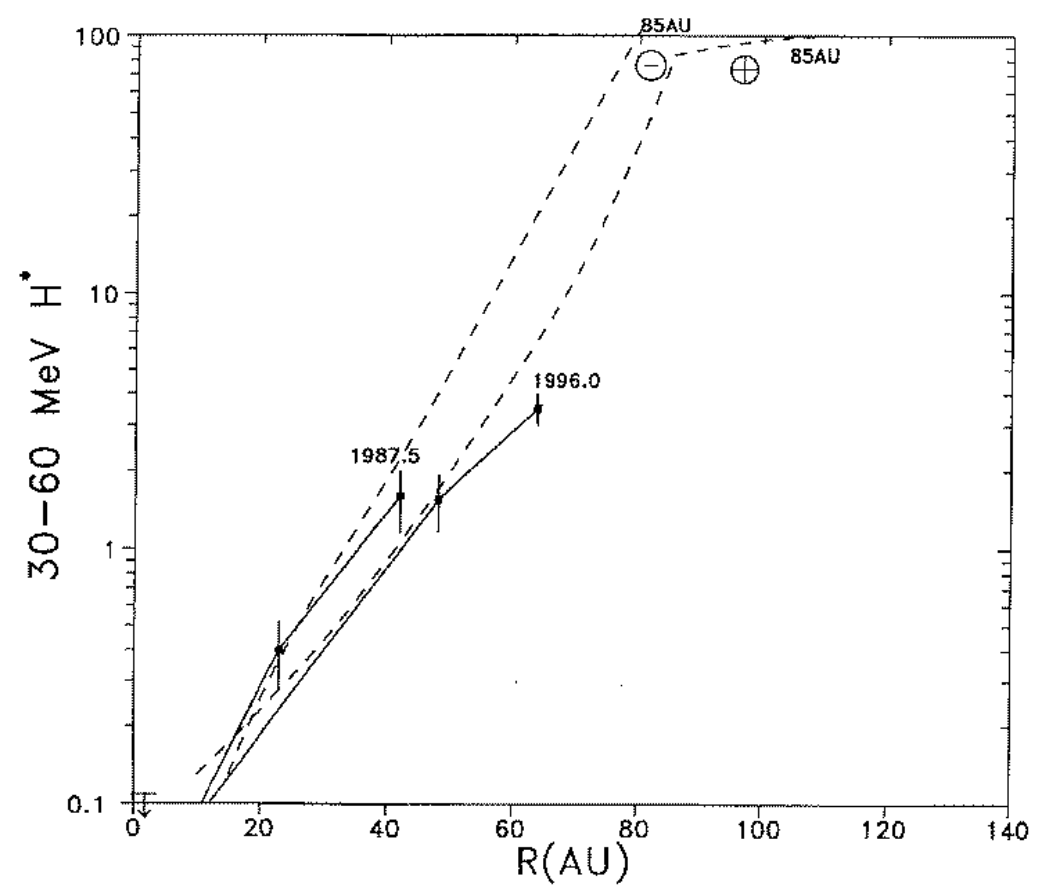

Figure 8. Same as Figure 6 but for $30-60 \mathrm{MeV} / \mathrm{n} \mathrm{H}^{+}$.

\subsection{Model Calculations}

Numerical solutions of the time-dependent cosmic ray transport equation (Equation 1) have been developed for a heliosphere containing a solar wind termination shock where first order Fermi acceleration of galactic cosmic rays (GCR) and lower energy particles (of the order of a hundred keV) takes place. Steenkamp (1995) developed a two-dimensional shock acceleration model that includes drift effects and a discontinuous (or continuous) shock transition in an axially symmetric heliosphere (model 1), while Le Roux et al. (1996) modeled a sphericallysymmetric heliosphere and a continuous solar wind termination shock (model 2). Both models are descendants of a unique steady-state acceleration model by Potgieter and Moraal (1988) which is still widely used, e.g., Stone et al. (1996).

Panels A and B of Figure 9 show anomalous cosmic ray (ACR) observations in 1987 at different heliocentric distances, compared to model 1, operated as an acceleration model with a source of anomalous particles on the SWTS (Steenberg and Moraal, 1996). The modelled ACR energy spectra, having a common form for different species, and their radial variations as a function of time, energy, and species are in excellent agreement with observations from the Voyager and Pioneer spacecraft. Panel C shows that the ACR component at the SWTS has a characteristic power law spectrum for energies up to a curvature cutoff energy. Models 1 and 2 are 


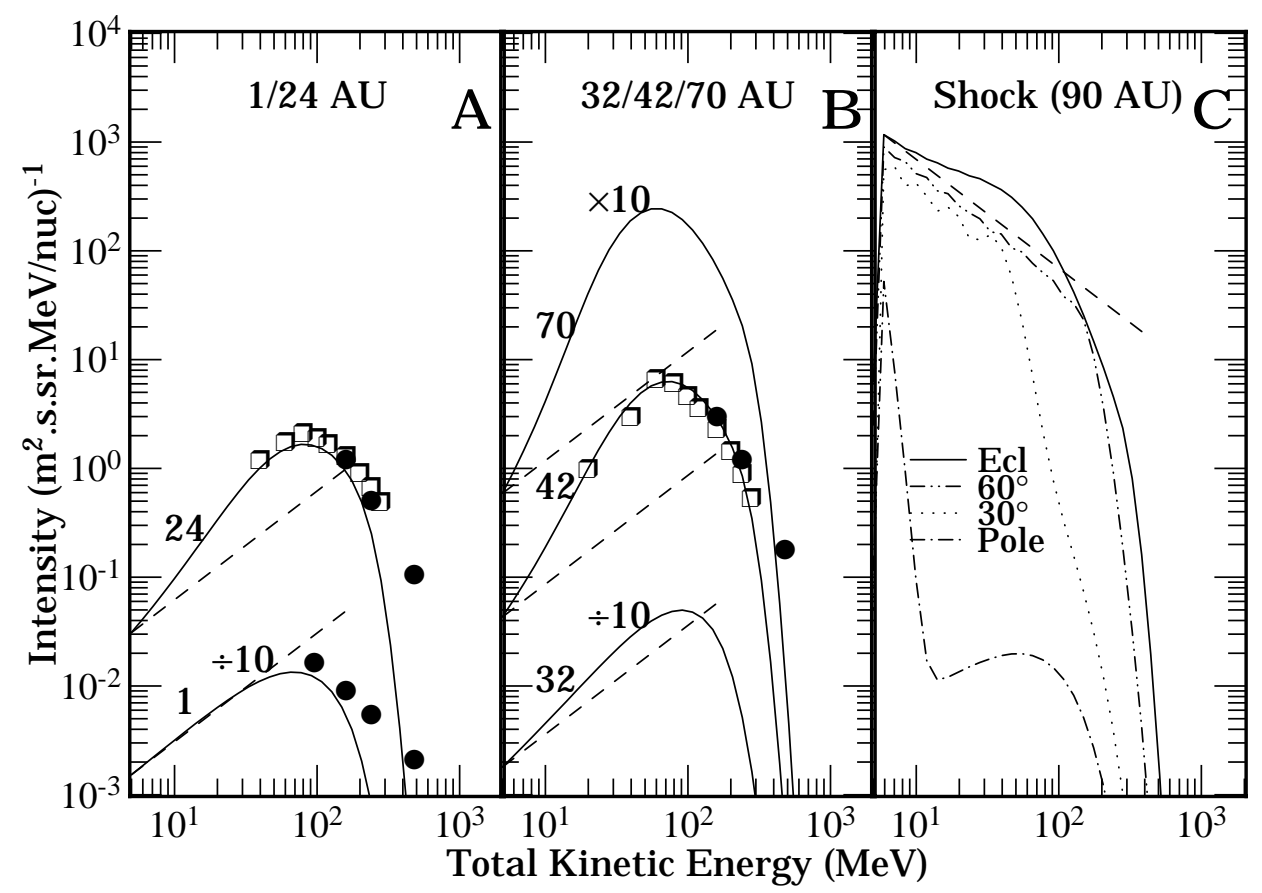

Figure 9. ACR spectra for a negative cycle showing the common spectral shape of ACR $\mathrm{He}^{+}$ and $\mathrm{O}^{+}$as a function of total kinetic energy. Panels $\mathrm{A}$ and $\mathrm{B}$ show $\mathrm{He}^{+}$(open squares) and $\mathrm{O}^{+}$ (closed dots) observations from IMP-8 (1 AU), Voyager 2 (24 AU), and Pioneer 10 (42 AU) in 1987 (from McDonald et al., 1992), together with modulated ACR spectra (solid curves) at the different spacecraft positions (Steenberg and Moraal, 1996). In Panel A differential spectra are shown at 1 AU (lower curve, intensity multiplied by $10^{-1}$ ) and $24 \mathrm{AU}$ (upper curve), in Panel B at $32 \mathrm{AU}$ (lower curve, multiplied by $10^{-1}$ ), at $42 \mathrm{AU}$ (centre curve, with observations) and at $70 \mathrm{AU}$ (upper curve, multiplied by 10). The straight dashed lines of the form $j \propto T$ are drawn through the lowest point of each calculated spectrum to represent the adiabatic limit. Panel $C$ shows drift-modified power law spectra for the $q A<0$ cycle, up to the so-called curvature cutoff energy, for four positions at the SWTS. The dashed line has the form $T^{-1}$, as expected for a strong planar shock.

able to explain the characteristics of the singly charged ACR species $\left(\mathrm{He}^{+}, \mathrm{O}^{+}\right.$and $\mathrm{N}^{+}$) which are easily distinguishable from the fully ionized galactic component (e.g. Steenkamp and Moraal, 1993a). The more complex case of protons, with only one charge state, was considered by Steenberg and Moraal (1995) and Le Roux et al. (1996), who used models 1 and 2 respectively, to investigate the spectral characteristics of anomalous protons during the 1987 solar minimum $(q A<0$ cycle). They concluded that the coincident spectral peaks of the ACR and GCR hydrogen combined with the greater radial gradient of the ACR component should make detection of this component exceedingly difficult inside a heliocentric radius of 20 to $30 \mathrm{AU}$, while it should be dominant in the outer heliosphere. Potgieter (1995) pointed out that the likelihood of detection in the inner heliosphere should be higher during the $q A>0$ cycle because of a shift to lower energies in the spectral peaks of the anomalous proton spectra. 
The so-called hysteresis effect in cosmic rays, where cosmic ray intensities lag behind the actual modulation conditions in the heliosphere, was investigated by Steenkamp and Moraal (1993b), Steenkamp (1995) and Steenberg and Moraal (1996). It was found that a pure modulation model (without a SWTS) produced the smallest hysteresis effect or temporal phase lag (about 4 months), followed by a GCR re-acceleration model (just under a year), and the ACR acceleration model (2 years). The large phase lags for ACRs were attributed to the long characteristic acceleration time for the first order Fermi acceleration process.

Steenberg and Moraal (1997) showed that particle drifts greatly modify ACR spectra at the SWTS, causing a harder (softer) spectrum in the ecliptic and a softer (harder) spectrum in the polar regions in the $q A<0(q A>0)$ drift cycle. It turns out that these modifications are greatly parameter dependent and need intensive further study.

The current focus is on: (1) Explaining the 1994 to 1997 ( $q A>0$ ) spectral observations of ACR and GCR spectra in the outer heliosphere (see also Report of Working Group 3). (2) Using a two-dimensional version of model 2 to study the re-acceleration and modulation of galactic and Jovian electrons at the SWTS (Haasbroek et al., 1997). (3) Modeling the injection of thermal ions into the SWTS (see also the Report of Working Group 3).

\section{Charge Dependent Spatial Modulation of Cosmic Rays}

B. Heber, M. S. Potgieter, P. Ferrando, G. Wibberenz, P. Evenson and J. R. Jokipii

\subsection{INTRODUCTION}

The comparison of the electron modulation with that of ions certainly provides the best information about the importance of modulation processes, such as heliospheric drifts, which depend on the sign of the particle charge. Garcia-Munoz et al. (1986) have clearly shown that the He/electron ratio at $\sim 0.8 \mathrm{GV}$ has been rapidly changing during the change of polarity of the solar magnetic field in the '70s and '80s (see also Evenson, 1998).

Ulysses was launched at the end of the $q A<0$ magnetic polarity period of the solar cycle, was in the ecliptic during the field reversal, and made its out-of-ecliptic journey on the first solar orbit during the $q A>0$ magnetic polarity. On board Ulysses, the Kiel Electron Telescope has provided a continuous record of cosmic ray fluxes, both for protons and helium from about $5 \mathrm{MeV} / \mathrm{n}$ up to several $\mathrm{GeV} / \mathrm{n}$ in different energy windows, and electrons from about $3 \mathrm{MeV}$ to several $\mathrm{GeV}$ (Simpson et al., 1992; Rastoin et al., 1996; Ferrando et al., 1996). In contrast to ion measurements, no high-energy $1 \mathrm{AU}$ electron data are available, so that it is not possible to disentangle temporal and spatial variations of the galactic electron fluxes. 


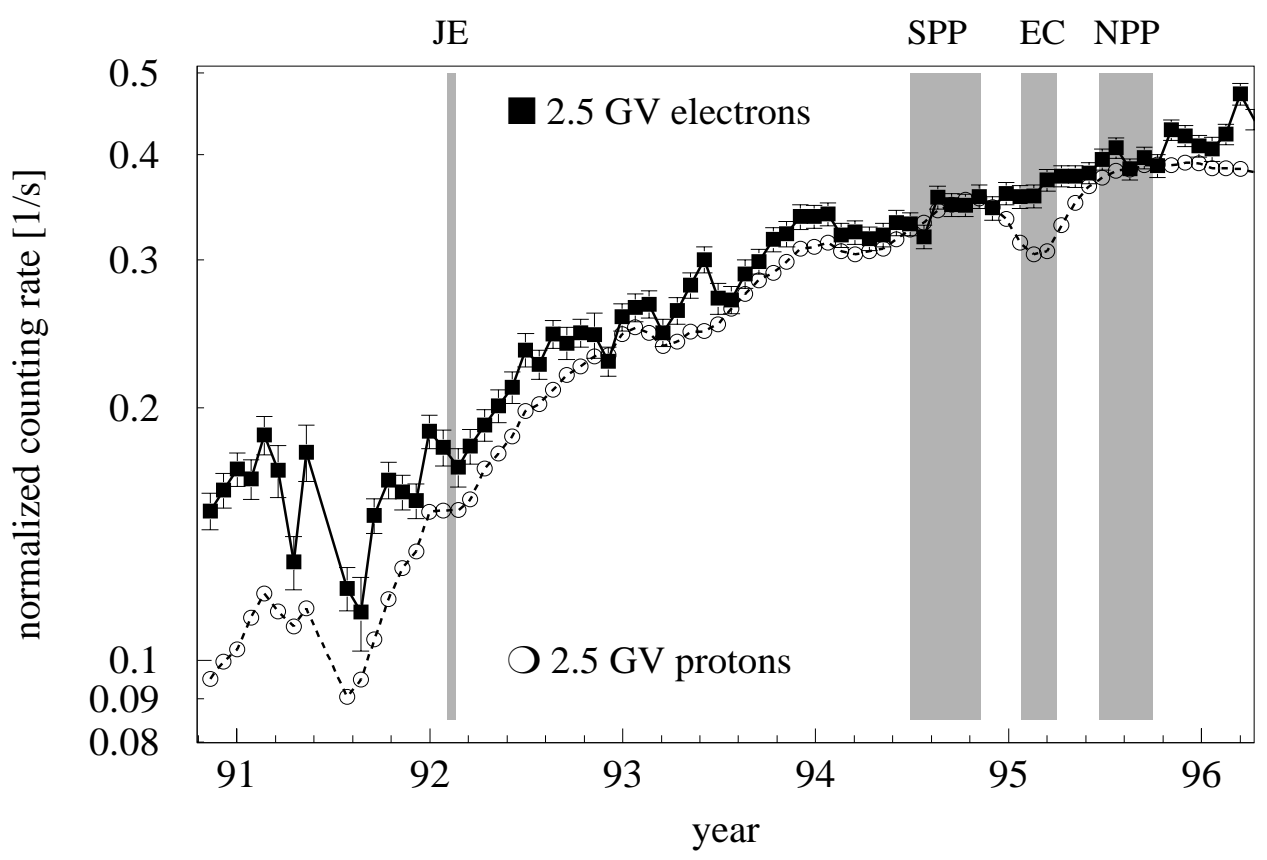

Figure 10. 26-day averaged quiet time counting rates of $2.5 \mathrm{GV}$ protons and electrons measured by the KET on Ulysses between late 1990 and March 1996. The rates have been normalised at the south polar pass (SPP). The Jovian encounter (JE), the equatorial crossing (EC) and the time periods of the south and north polar passes (NPP) are marked.

Figure 10 displays the 26-day averaged quiet time KET counting rates of 250$2200 \mathrm{MeV}$ protons and $900-4600 \mathrm{MeV}$ electrons. (The 38-125 MeV proton channel, not shown here, has been used to determine these quiet time periods when the galactic cosmic ray flux was not contaminated by solar or locally accelerated energetic particles.) The time-profiles of both channels mix temporal and spatial (radial and latitudinal) variations. Electrons and protons show qualitatively similar features with a general solar cycle recovery. There are two periods during which the electron and proton profiles show significant charge dependent effects: during the time period of the pole to pole passage in 1994/1995 and during the reversal of the heliospheric magnetic field (HMF) in 1991.

Two aspects of the galactic electron to proton ratio (e/p) are discussed here: first, the determination of the spatial charge-dependent modulation during solar minimum conditions and second, the evolution of this ratio as a function of the HMF polarity.

\subsection{SPATIAl VARIATION OF THE E/P RATiO}

As is apparent in Figure 10, the $2.5 \mathrm{GV}$ electron and proton time-profiles differ during the Ulysses fast latitude scan. The proton profile has a ' $\mathrm{V}$ ' shape during 

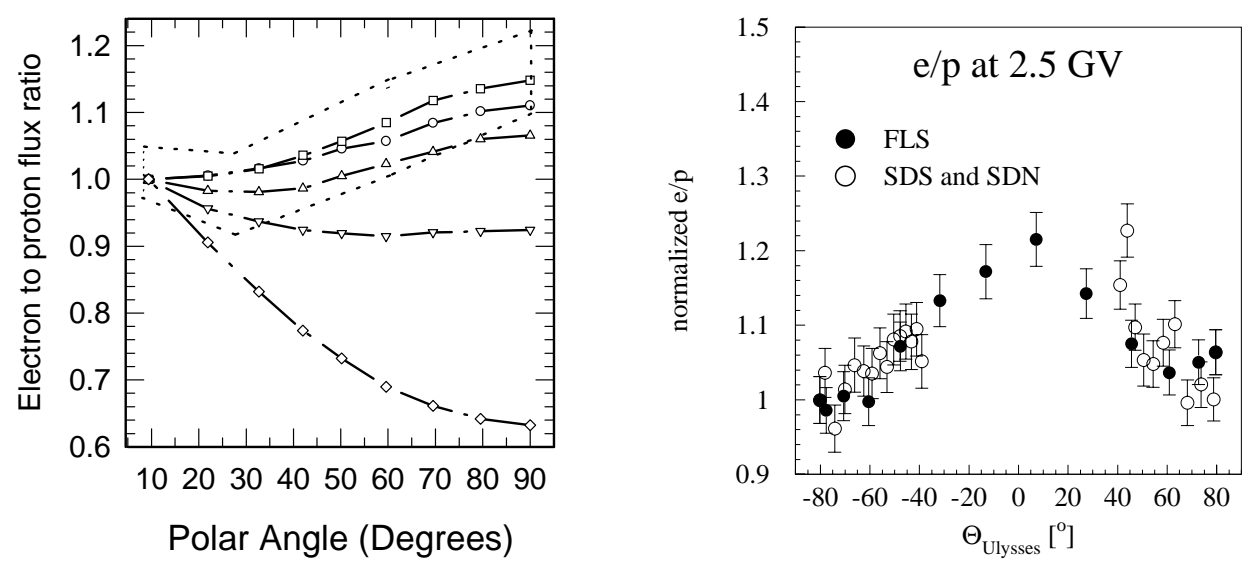

Figure 11. Left panel: Computed e/p flux ratio for $q A>0$ cycle between pole and equator as a function of polar angle $\left(0^{\circ}\right.$ is pole, $90^{\circ}$ equator, from Potgieter et al., 1997). $5 \mathrm{GV}$ : circles; $1 \mathrm{GV}$ : squares; $500 \mathrm{MV}, 300 \mathrm{MV}$ and $100 \mathrm{MV}$ : three bottom curves respectively. The dotted "box" represents the maximum KET data range $(0.9-2.5 \mathrm{GV})$.

Right panel: e/p ratio measured by KET as a function of Ulysses heliographic latitude $\Theta$ for $2.5 \mathrm{GV}$ particles (FLS: fast latitude scan; SDS and SDN: slow descent south and north).

that period (early 1995), with a flux minimum close to the equator (see McKibben, 1998) whereas the electron profile is steadily increasing. The electron and proton rates, normalized at the south pole, reach the same level (within error bars) at the north pole. This indicates with high probability that electrons and protons had the same relative (small) recovery during that period.

The right panel of Figure 11 shows the variation of the e/p-ratio at $2.5 \mathrm{GV}$ as a function of heliographic latitude. [The full circles are data taken during the fast latitude scan, while open circles are data taken during the slow ascent to the south polar region in 1993 and 1994 and during the slow descent from the north pole in 1995 and 1996. The earliest (latest) point, at $\sim 40^{\circ} \mathrm{S}\left(40^{\circ} \mathrm{N}\right)$, corresponds to data collected in Sep. 1993 (Mar. 1996)]. The $\sim 20 \%$ difference between highest latitudes and equator is entirely due to the proton latitudinal gradient, as is the flattening of this ratio above $60^{\circ}$. Electrons have a homogeneous latitudinal distribution (Ferrando, 1997). An important point is that the data taken during the slow latitude descents are consistent with the measurements made during the fast latitude scan. This is remarkable because the galactic cosmic ray proton and electron fluxes increase from 1993 to 1996 and the radial distance from the Sun during the fast latitude scan (2.3 to $1.3 \mathrm{AU}$ ) is smaller than in the other periods (2.0 to $4.7 \mathrm{AU})$. Within the uncertainty of the data, the amplitude of the e/p-variation as a function of rigidity reflects the rigidity dependence of the proton latitudinal gradient (see also Figure 4).

The left panel of Figure 11 shows the calculated latitudinal dependence of the e/p ratio in the inner heliosphere for five different rigidities (Potgieter et al., 1997). Because of drift effects the computed ratio at rigidities above $0.5 \mathrm{GV}$ shows, 
in agreement with the observations, a maximum at the heliospheric equator and minima in the polar regions in the inner heliosphere. The steady-state modulation model used by Potgieter et al. (1997) includes all major modulation mechanisms. Steady-state is justified because solar minimum conditions prevailed during the Ulysses fast latitude scan. For the more general predictions of time-dependent models, see Haasbroek et al. (1995), and Ferrando et al. (1996). For a general description of model predictions, see Potgieter (1998).

In a $q A<0$ solar cycle (e.g. 1980 to 1991) protons drift along the heliospheric current sheet into the inner heliosphere and electrons move outwards along the current sheet. In a simple picture one should expect lower proton (higher electron) fluxes in polar regions. Therefore the corresponding e/p pole to equator ratio should be greater than one. As the right panel of Figure 3 in Potgieter (1998) shows, this is also expected from advanced modulation modelling for rigidities above several hundred MV in the outer heliosphere. In the inner heliosphere the increase of the $\mathrm{e} / \mathrm{p}$ pole to equator ratio becomes small. However, with Ulysses we would have the unique opportunity to measure the e/p ratio in a $q A<0$ solar cycle close to solar minimum conditions during a third fast pole-to-pole passage in 2006. Such measurements would provide crucial information for our understanding of spatial charge dependent modulation under solar minimum conditions

At lower rigidities protons but not electrons are mainly modulated by adiabatic cooling. To investigate the charge-dependent modulation at lower rigidities $(<$ several hundred MV) it is necessary to measure the positron intensity as a function of latitude. The KET does not separate positrons and electrons.

\subsection{The E/P RATIO DuRING THE HMF REVERSAL}

The relative $2.5 \mathrm{GV}$ electron and proton fluxes show an important variation from late 1990 to the beginning of 1992, i.e., in the time period of the HMF polarity reversal (shaded area in Figure 12). The e/p ratio decreases from about 1.6 to 1.2, approximately twice the spatial variation observed during the fast latitude scan.

As discussed by Ferrando (1997), this 1991 decrease might reflect a time lag for the recovery of the electrons compared to the protons. The e/p values before and after this reversal are in fact consistent with being almost constant. Ferrando (1997) reports that the variation of the $0.9 \mathrm{GV}$ e/p ratio is very similar to the $\mathrm{He} / \mathrm{p}$ variations reported by Garcia-Munoz et al. (1986). It can thus be quantitatively concluded that the same phenomenon was observed two solar cycles apart, for the same magnetic field polarity reversal. One would expect such big changes in the e/p ratio if drifts dominate throughout the 11 year solar cycle. The dominance, even the occurrence, of drifts during solar maximum periods has however been questioned (Potgieter, 1993; 1995; Le Roux and Potgieter, 1995). It is at present not clear what causes these large charge-dependent effects during solar maximum activity epochs (see also Evenson, 1998; Potgieter, 1998). A direct causal link between the HMF structure and the e/p ratio appears to be attractive, but further studies are obviously 


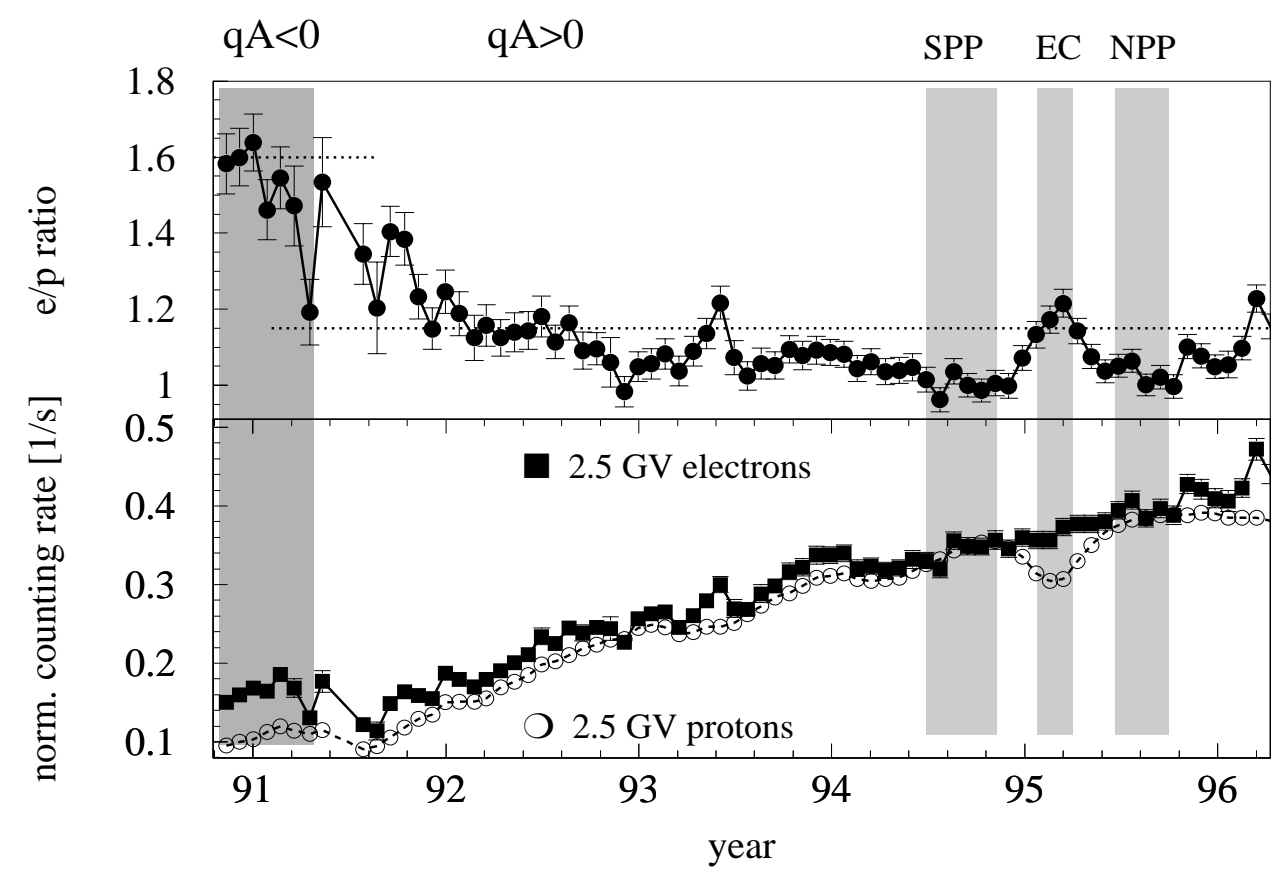

Figure 12. 26-day averaged quiet time $2.5 \mathrm{GV}$ electron and proton fluxes and e/p ratio during the time period from late 1990 to March 1996.

necessary before settling this issue. The next HMF reversal is not expected to occur before 2001. By that time Ulysses will be at high northern heliospheric latitudes, allowing us for the very first time to investigate a HMF reversal as a function of latitude.

\section{Implications of 26-Day Variations for the Global Modulation}

M. Zhang, J. A. Simpson, H. Kunow, J. R. Jokipii, M. S. Potgieter, R. A. Burger and A. C. Cummings

26-day recurrent variations in the fluxes of cosmic rays and anomalous components are generated by solar wind and interplanetary magnetic field structures that recur with the period of solar rotation. This subject is mainly described in the report of Working Group 2. Although 26-day recurrent variations (sometimes called 27-day variations when observed from an Earth-orbiting spacecraft or a ground-based instrument) have been studied for more than 40 years, it is not until the recent Ulysses observations in the polar regions of the heliosphere that we have realized their importance for understanding the global modulation of cosmic rays.

Recurrent structures in the interplanetary medium during the solar activity minima mainly appear in low and middle latitude regions. There are two basic kinds 
of recurrent structures: corotating interaction regions (CIRs) and the heliospheric current sheet (HCS). Studying recurrent variations of cosmic ray fluxes in their relation to the CIRs or the HCS at low latitudes can provide considerable information on the mechanisms by which cosmic rays transport, e.g., diffusion and gradient/curvature drifts, and thereby, indirectly, information on solar modulation in the global heliosphere. Although CIRs cannot produce a global or a long term effect on the cosmic rays (e.g., Kóta and Jokipii, 1991; Potgieter and Le Roux, 1994), studies using magnetic field and cosmic ray measurements from Voyager in the outer heliosphere (Burlaga et al., 1985) have established that the reduced particle diffusion in the enhanced magnetic fields inside the interaction regions is a source for cosmic ray depressions. Based on this observation, it has been proposed that the 11-year solar cycle cosmic ray variation is the result of accumulated disturbances from global merged interactions (Burlaga et al., 1993; Le Roux and Potgieter, 1995).

Recently, Zhang (1997) pointed out that there is a linear relationship between the amplitude of 26-day recurrent variations and the magnitude of latitude gradients in the fluxes of both galactic cosmic rays and the anomalous component (Figure 13, as an example). The linear relationship holds for recurrent variations observed at all latitudes and radial distances in the inner heliosphere. This relationship means that particles which have large latitude gradients are also strongly modulated by corotating structures regardless of their charges and energies. In other words, it implies that particles of different energies and charges have approximately similar distributions as a function of latitude and longitude. Zhang's investigation with Voyager and IMP-8 measurements in the previous solar cycle, when the solar magnetic field polarity was reversed, also displayed this linear relationship even though the latitude gradient had a negative sign.

The linear relationship provides a direct connection between modulation by corotating structures and that by the global heliosphere. It suggests that there may be a common mechanism that controls both flux variations in recurrent modulation, as well as the latitudinal flux distribution. This common mechanism can result from either the heliospheric magnetic field or from some aspect of the modulation process.

A single interplanetary magnetic field structure that can control both global latitudinal and longitudinal cosmic ray flux distributions can account for a correlation between the latitude gradient and longitudinal recurrent variations. Such a structure, however, is not expected to be the CIRs alone since they should not have a global effect, particularly during the present solar cycle of magnetic polarity $q A>0$. Here, positively charged cosmic ray particles come from the polar regions of the heliosphere, and CIRs at low latitudes should not have a significant effect on the polar flux level. An alternative explanation could involve the HCS, which could have an effect on the global heliospheric cosmic ray distribution, as well as on recurrent modulation. In fact, even before Ulysses made its observations, this idea was proposed by Stone and Cummings (Stone, 1987; Cummings 


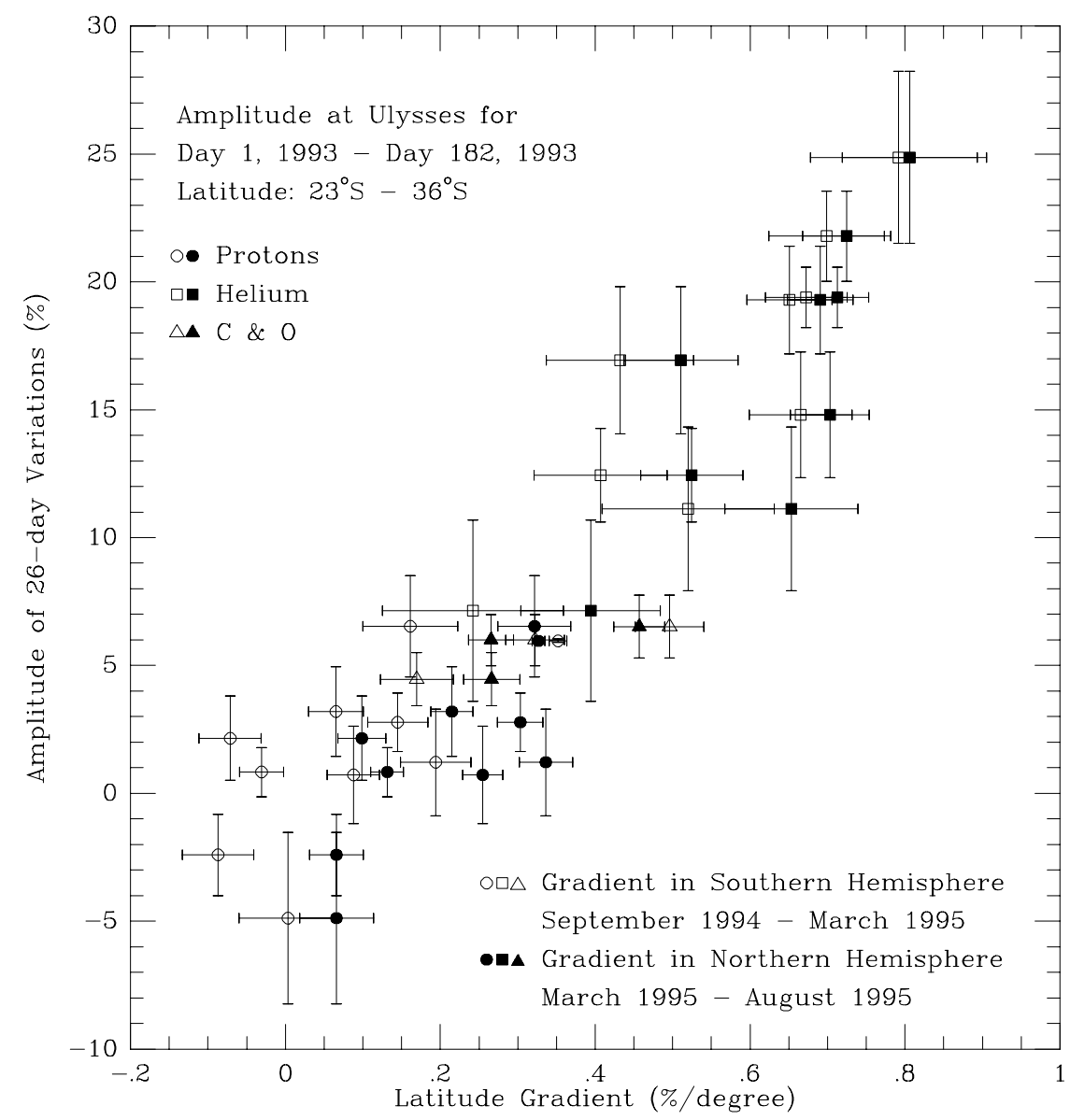

Figure 13. A linear relationship between the latitude gradient and the amplitude of 26-day variations is seen by Ulysses. Each data point corresponds to a particle channel. The amplitudes were calculated from data measured in a sample period at middle latitudes in 1993 when the recurrent variations were largest due to strong compression of the solar wind and magnetic fields and much greater than the errors due to statistical fluctuations. The gradients were calculated from data obtained during the south pole to north pole fast latitude scan period. The correlation between the amplitude of the recurrent variations and the latitude gradient is independent of the time period studied.

and Stone, 1988), who argued that cosmic ray fluxes are roughly organized by heliomagnetic latitude, a quantity proportional to the distance to the HCS, and that periodic changes in the heliomagnetic latitude at a spacecraft can simulate 26-day recurrent flux variations. In this theory, the linear relationship between the latitude gradient and the amplitude of recurrent variations is automatically satisfied if the 2-dimensional longitude-latitude flux distribution maps have a similarity among particles of different energies and charges. However, it remains to be answered whether the observed 26-day recurrent variations can be produced by the HCS. Zhang et al. (1995) found no correlation between the phase of 26-day cosmic ray 
recurrent variations and heliomagnetic latitude, and Simpson (1998) pointed out that the amplitude of 26-day variations at the same heliocentric latitude did not change significantly even though the tilt angle of the HCS changed from $30^{\circ}$ in 1993 to $15^{\circ}$ in 1996 while the latitude gradient remained the same. Rather, these studies, along with other studies of accompanying low-energy particle recurrent events (Sanderson et al., 1995; Roelof et al., 1996), seem to favor CIRs as a modulation source for the recurrent variations. Further studies are needed to determine the relative contribution of CIRs and the HCS to the recurrent variations.

If the recurrent variations and the latitude gradients are determined by different interplanetary magnetic fields or even different particle transport mechanisms (i. e., the recurrent variations are caused by the compressed magnetic fields of CIRs and the latitude gradients are determined by the global large- and small-scale interplanetary magnetic fields from the poles to the equator), then the correlation requires that there must be some common aspect of the modulation process controlling the flux variations in these phenomena. Zhang (1997) argues that this common aspect is the effect of adiabatic deceleration by the expanding solar wind. As cosmic ray particles are transported from polar regions to the equator, they must continuously undergo adiabatic energy loss, thus resulting in lower fluxes along their path. Similarly, the flux decreases caused by the compressed magnetic fields of CIRs are the result of adiabatic deceleration of the particles trapped behind the strong fields (Thomas and Gall, 1984). The latitude gradients and the amplitudes of recurrent variations are both a deviation in the cosmic ray flux caused by a perturbation in the modulation experienced by the cosmic rays in different locations or in different interplanetary conditions. Thus, the latitude gradients and the recurrent amplitudes should be determined by the amount of additional energy loss they suffer due to this extra modulation, as well as by the shape of their spectra near the location of observation (the shape of the spectrum determines how the flux varies with energy changes). If the amount of energy loss as a function of particle rigidity are similar (which may more or less be true) in the particle transport processes for these two phenomena, then the ratio of the latitude gradient and the amplitude of recurrent variations is left independent of particle rigidity, consistent with the correlation in Figure 13. With similar arguments, this explanation predicts that the radial gradient of cosmic ray fluxes should also roughly have a linear relationship to the latitude gradient.

The polar regions of the heliosphere beyond approximately $40^{\circ}$ south and north latitude are dominated by fast steady solar wind streams from polar coronal holes (Phillips et al., 1995). There, the large-scale average magnetic field is remarkably uniform and shows no signatures of the solar magnetic dipole (Balogh et al., 1995). It was, therefore, a surprising discovery that recurrent variations of cosmic rays and anomalous components extend to the Ulysses maximum solar polar latitude of $80^{\circ}$ south and north (Simpson et al., 1995; Simpson, 1998, and references therein). Autocorrelation studies revealed that the recurrent period in the polar regions is 26 days, the same as the equatorial rotation rate. Since the solar rotation period in the 
polar region is much longer ( $\sim 32$ days) due to the differential rotation, the recurrent variations of cosmic ray fluxes must come from low latitudes. Thus the recurrent variations become a large scale global heliospheric modulation phenomenon.

Since recurrent modulation source regions occur only at low latitudes, it was immediately recognized that there must be efficient particle transport in latitude. The gradient/curvature drifts cannot carry recurrent variations from low latitudes to the polar regions because in the present cycle of solar magnetic polarity $(q A>0)$ positively charged particles drift from the poles to the equator. Therefore the only way for the recurrent variations to propagate to high latitudes is through cross-latitudinal diffusion $\left(\kappa_{\theta \theta}\right)$. Kóta and Jokipii (1995) have generated computer models that yield recurrent variations at high latitudes under the assumption of large $\kappa_{\theta \theta}$. These models are consistent with the small latitude gradients observed from Ulysses (Simpson et al., 1995; Potgieter et al., 1997).

The remaining question is how to achieve a sufficiently large $\kappa_{\theta \theta}$. Two approaches have been proposed: Kóta and Jokipii (1995) suggested a mechanism of random walking of magnetic field lines across latitude to increase the perpendicular diffusion coefficient in the $\theta$ direction. A different approach was developed by Fisk (1996), who pointed out that the interplay between the differential rotation of the footpoints of the magnetic field lines on the photosphere and the subsequent non-radial expansion of the field with the solar wind plasma from rigidly-rotating coronal holes may result in extensive excursions of heliospheric magnetic fields lines over a wide range of heliographic latitudes. A spacecraft at high latitudes is then periodically connected to a remote CIR at low latitudes. Particle diffusion along the direction parallel to the average magnetic field gives rise to particle transport in latitude. Detailed discussions of these models and their tests are presented in the report of Working Group 2.

\section{Summary}

The previous five sections each illustrate an important aspect of the global processes which affects galactic cosmic ray modulation at and around solar minimum conditions; together they lead to the following general conclusions:

1. There is no evidence of any deficiencies in the basic Equation (1) which describes the behavior of the cosmic rays in the solar wind. Admittedly, there is still a wide variation in the choice of the parameters in this equation. However, within acceptable ranges for these parameters, the observed phenomena can be accounted for.

2. The overall flow patterns of cosmic ray ions in the heliosphere, as determined by drift effects, appear to be understood. In the current cycle, the recovery of ions in the inner heliosphere precedes that in the outer heliosphere, as would be expected if the access of the ions is primarily over the solar poles, consistent with that predicted by drift effects. Conversely, in the previous 
cycle, the recovery is faster in the outer heliosphere as if the access is along the equatorial current sheet, again consistent with drift effects.

3. The radial gradients near solar minimum are also, in general, consistent with drift effects. The gradients tend to be smaller in the current cycle when access is easier over the poles, and larger in the previous cycle when the access is along the current sheet. It is important in making this evaluation to consider the radial gradients on a large spatial scale; smaller scale variations can distort the expected variation between solar minima.

4. The small latitude gradients in cosmic rays observed by Ulysses, as well as the observation that particles accelerated in or modulated by low latitude corotating interaction regions propagate easily to high latitudes, demands that cross-field transport, at least in the heliocentric polar direction, is quite easy. The mechanism by which this transport occurs, whether by cross-field diffusion or by direct magnetic connection, is uncertain.

5 . The offset of the plane of symmetry for modulation by $10^{\circ}$ towards the southern hemisphere, as observed by Ulysses, has no definitive explanation. The observation appears to suggest an asymmetry between the solar hemispheres; however, there is no direct evidence for such an asymmetry in the magnetic field or plasma observations.

6. The model in which anomalous cosmic rays are accelerated at the termination shock of the solar wind, and subsequently propagate in a standard model for galactic cosmic ray modulation, is consistent with many of the observed features of the anomalous component. The faster recovery in the inner heliosphere during the current approach to solar minimum, as opposed to in the previous cycle, is consistent with propagation theories in which drift effects are important. The difference between solar cycles, however, is too large to be due only to drifts within the heliosphere and may require a solar magnetic cycle difference in the latitudinal distribution of the anomalous component at the termination shock. Such a difference can also result from drift effects, since the direction in which particles drift along the shock front varies with the solar magnetic cycle.

7. The electron observations, as a test of our understanding of cosmic ray modulation, are inconclusive. Drift effects suggest that electrons and ions should behave substantially differently; indeed, the electrons should exhibit behavior similar to the ions in the previous or following cycle. Electron observations, however, have not been made over multiple solar cycles with the same fidelity as those of ions, and there can be confusion as to the extent to which the galactic electron spectrum contains galactic positrons. Moreover, the principal test of the electron vs. ion behavior should occur near the reversal of the polarity of the heliospheric magnetic field. However, this occurs just following solar maximum conditions when it is unclear whether the field is sufficiently ordered to experience the full effects of drifts. 
8. No single model, with a single choice for the parameters used in Equation (1), has been able to account for all the observed features of galactic and anomalous cosmic ray behavior - time and spatial variations, spectra, electron and ion behavior. In part, this may reflect that the fact that even with the many advances in numerical solutions to Equation (1), a full time-dependent, threedimensional code does not exist. It may also reflect the fact that the best choices for the parameters, driven by the micro-physics of the interactions of energetic particles with the heliospheric magnetic field, has yet to be found.

\section{Note Added at Press Time: North-South Asymmetry in the Heliosphere}

There has been a significant amount of attention given at this workshop to the interpretation of the north-south asymmetry observed by Ulysses in the cosmic-ray flux (see McKibben, 1998). This problem went without clear resolution during the workshop, primarily because the most-straightforward interpretation of the observations seemed to be at variance with magnetic-field observations. In their original paper reporting on their observations of the apparent latitudinal asymmetry in the cosmic-ray counting rate, Simpson et al. (1996) stated among other things, that "the plane of symmetry is offset southward from the Sun's heliographic equator by $\approx 10^{\circ}$ of latitude". They also pointed out the importance of the fact that the latitudinal gradients were the same in the northern and southern hemispheres, and that the cosmic-ray intensity near the north pole exceeded by $10 \%-15 \%$ that near the solar pole, consistent with a global shift. A similar effect was reported by Heber et al. (1996b). One way to change the plane of symmetry, in the framework of the current picture of cosmic-ray modulation, is to shift the current sheet some $10^{\circ}$ south.

This observation was discussed in some detail at the workshop and it was concluded that it could not be consistent with the magnetic field observed at Ulysses. The point being that Maxwell's equations require that the total radial magnetic flux out of the sun be zero. If the current sheet is shifted toward one pole, then the hemisphere toward which the current sheet is shifted must have a radial magnetic field magnitude which is larger. The average field in the southern hemisphere would be a factor of 1.3 larger than that in the northern hemisphere, for the shift of $7^{\circ}$ suggested by Simpson et al. (1996), to be present. The initial response of the magnetometer team to this was that such a large a change in the magnetic field was not observed. In the face of this conclusion, other alternatives were discussed, including a new magnetic-field configuration and warps in the current sheet. But none of these seemed very compelling, and the problem was temporarily dropped.

However, in the past 2 months, a new look at the magnetic-field data seems to have resolved this problem. As reported in a talk by Jokipii at the Fall 1997 AGU meeting in San Francisco (Smith et al., 1997), the Ulysses magnetic-field data 
actually do show a roughly $30 \%$ change in the radial component of the magnetic field roughly consistent with in-ecliptic observations carried out on the WIND spacecraft.

The problem in relying solely on the Ulysses measurements is that the spacecraft was basically in one magnetic sector at a time, a negative sector when in the south and a positive one when in the north. So it was not possible to compare the radial component inthe two sectors, simultaneously, using Ulysses alone. When this limitation was recognized, the WIND data, obtained near the heliospheric equator, were examined to see how the radial fields compared in the two sectors as they swept past WIND. Unexpectedly, a significant difference between the inward and outward sectors was observed throughout the interval of the Ulysses fast latitude scan, and whose magnitude is consistent with the southward displacement of the heliospheric current sheet inferred from the north-south asymmetry in the cosmic rays. The WIND data show that this difference gradually disappeared toward the end of the interval so that when Ulysses passed into the north hemisphere the radial component there was essentially the same as it had been when Ulysses was in the south hemisphere. Thus, the time dependence had obscured the evidence of a difference in the two sectors as seen at Ulysses. That a time dependence can masquerade as a spatial dependence is well known. However, in this instance, the opposite effect occurred with a time dependence actually compensating for a spatial dependence.

In the same AGU presentation, Smith et al. (1997) presented preliminary results from two-dimensional simulations of the modulation of galactic cosmic rays, using the Arizona modulation code, which verified that cosmic-ray asymmetries similar to those observed by the cosmic-ray experiments are a natural result of the observed asymmetry in the magnetic field. Such a model would also increase the intensity at the North pole. Hence, the asymmetry problem appears to have a straightforward resolution. The results of Smith et al. (1997) will be incorporated into a publication in the near future.

\section{Acknowledgements}

One of the authors (KPW) wishes to acknowledge the support of Ms. C. Nilsson in producing this report.

\section{References}

Balogh, A., Smith, E. J., Tsurutani, B. T., Southwood, D. J., Forsyth, R. J. and Horbury, T. S.: 1995, 'The Heliospheric Magnetic Field Over the South Polar Region of the Sun', Science 268, $1007-1010$.

Balogh, A.: 1998, Space Science Rev., this volume.

Burlaga, L. F., McDonald, F. B., Goldstein, M. L. and Lazarus, A. J.: 1985, 'Cosmic ray modulation and turbulent interaction near 11 AU', J. Geophys. Res. 90, 12,027-12,039. 
Burlaga, L. F., McDonald, F. B. and Ness, N. F.: 1993, 'Cosmic ray modulation and the distant heliospheric magnetic field: Voyager 1 and 2 observations from 1986 to 1989', J. Geophys. Res. 98, $1-11$.

Cummings, A. C., Stone, E. C. and Webber, W. R.: 1987, 'Latitudinal and radial gradients of anomalous and galactic cosmic rays in the outer heliosphere', Geophys. Res. Lett. 14, 174-177.

Cummings, A. C. and Stone, E. C.: 1988, 'Composition, gradients and temporal variations of the anomalous cosmic ray component', Proc. Sixth Intl. Solar Wind Conf., NCAR Tech. Note, 306, 599, Natl. Center for Atoms. Res., Boulder, Colo.

Cummings, A. C., Mewaldt, R. A., Stone, E. C. and Webber, W.R.: 1990, 'Radial and latitudinal gradients of anomalous cosmic-ray oxygen and helium from 1 to 41 AU', Proc. 21st Int. Cosmic Conf. (Adelaide), 6, 206-209.

Cummings, A. C., Mewaldt, R. A., Blake, J. B., Cummings, M., Franz, M, Hovestadt, D., Klecker, B., Mason, G. M., Mazur, J. E., Stone, E. C., von Rosenvinge, T. T. and Webber, W. R.: 1995, 'Anomalous cosmic ray oxygen gradients throughout the heliosphere', Geophys. Res. Lett. 22, 341-344.

Cummings, A. C. and Stone, E. C.: 1998, Space Science Rev., this volume.

Evenson, P.: 1998, Space Science Rev., this volume.

Ferrando, P.: 1997, 'Mev to GeV electron propagation and modulation: Results of the KET telescope onboard Ulysses', Adv. Space Res. 19(6), 905-915.

Ferrando, P., A. Raviart, L.J. Haasbroek, M.S. Potgieter, W. Dröge, B. Heber, H. Kunow, R. MüllerMellin, H. Sierks, G. Wibberenz, and C. Paizis: 1996, 'Latitude Variations of $\sim 7 \mathrm{MeV}$ and $>300 \mathrm{MeV}$ Cosmic Ray Electron Fluxes in the Heliosphere: Ulysses COSPIN/KET Results and Implications', Astron. Astrophys. 316, 528-537.

Fillius, W.: 1989, 'Cosmic ray gradients in the heliosphere', Adv. Space Res. 9(4), 209-219.

Fisk, L.A.: 1996, 'Motion of the Footpoints of Heliospheric Magnetic Field Lines at the Sun: Implications for Recurrent Energetic Particle Events at High Heliographic Latitudes', J. Geophys. Res. 101, 15,547-15,554.

Fisk, L. A., Forman, M. A. and Axford, W. I.: 1973, 'Solar modulation of galactic cosmic rays 3. Implications of the Compton-Getting coefficient', J. Geophys. Res. 78, 995-1006.

Forsyth, R. J., Balogh, A., Smith, E. J., Murphy, N. and McComas, D. J.: 1995, 'The underlying magnetic field direction in Ulysses observations of the southern polar heliosphere', Geophys. Res. Lett. 22, 3321-3324.

Forsyth, R.J., Balogh, A., Horbury, T. S., Erdös, G., Smith, E. J. and Burton, M. E.: 1996, 'The Heliospheric Magnetic Field at Solar Minimum: Ulysses Observations from Pole to Pole', Astron. Astrophys. 316, 287-295.

Fujii, Z. and McDonald, F. B.: 1997, 'Radial intensity gradients of galactic cosmic rays (1972-1995) in the heliosphere', J. Geophys. Res. 102, 24,201-24,208.

Garcia-Munoz, M., Meyer, P., Pyle, K. R. and Simpson, J. A.: 1986, 'The dependence of solar modulation on the sign of the cosmic ray particle charge', J. Geophys. Res. 91, 2858-2866.

Giacalone, J.: 1998, Space Science Rev., this volume.

Gleeson, L. J. and Axford, W. I.: 1967, 'Cosmic rays in the interplanetary medium', Astrophys. J. 149, L115.

Gleeson, L. J. and I. H. Urch: 1971, 'Energy loss and modulation of galactic cosmic rays', Astrophys. Space Sci. 11, 288.

Goldstein, M. J., Fisk, L. A. and Ramaty, R.: 1970, 'Energy loss of cosmic rays in the interplanetary medium', Phys. Rev. Lett. 25, 832.

Haasbroek, L., Potgieter, M. and Le Roux, J. A.: 1995, 'The time-dependent recovery after the large cosmic ray decrease in 1991', Proc. 24th Int. Cosmic Ray Conf. (Rome), 4, 710-713.

Haasbroek, L. J., Potgieter, M. S. and Le Roux, J. A.: 1997, 'Acceleration of galactic and Jovian electrons at the heliospheric solar wind termination shock', Adv. Space. Res. 19(6), 953-956.

Hattingh, M., Burger, R. A., Potgieter, M. S. and Haasbroek, L. J.: 1997, 'Cosmic Ray Latitudinal Effects Predicted by a Three-Dimensional Drift Model' , Adv. Space Res. 19(6), 893-896.

Heber, B., Dröge, W., Ferrando, P., Haasbroek, L. J., Kunow, H., Müller-Mellin, R., Paizis, C., Potgieter, M. S., Raviart, A. and Wibberenz, G.: 1996a, 'Spatial Variation of $>40 \mathrm{MeV} / \mathrm{n}$ Nuclei Fluxes Observed during the Ulysses Rapid Latitude Scan', Astron. Astrophys. 316, 538-546. 
Heber, B, Dröge, W., Kunow, H., Müller-Mellin, R., Wibberenz, G., Ferrando, P., Raviart, A. and Paizis, C.: 1996b, 'Spatial variation of $>106 \mathrm{MeV}$ proton fluxes observed during Ulysses rapid latitude scan: Ulysses COSPIN/KET results', Geophys. Res. Lett. 23, 1513-1516.

Heber, B., Belov, A. V., Raviart, A., Paizis, C., Eroshenko, E. A., Yanke, V. G., Dröge, W., Green, G., and Röhrs, K.: 1997a, 'Latitudinal and Radial Variation of $>2$ GeV Protons Derived by Ulysses COSPIN/KET and Neutron Monitor Network Observations', Proc. 25th Int. Cosmic Ray Conf. (Durban), 2, 85-88.

Heber, B., Bothmer, V., Dröge, W., Kunow, H., Müller-Mellin, R., Sierks, H., Wibberenz, G., Ferrando, P., Raviart, A., Paizis, C., McComas, D., Potgieter, M. S., Burger, R. A., Hattingh, M. and Haasbroek, L. J.: 1997b, 'Latitudinal Distribution of $>106 \mathrm{MeV}$ Protons and Its Relation to the Ambient Solar Wind in the Inner Southern and Northern Heliosphere: Ulysses COSPIN/KET Results', J. Geophys. Res., in press.

Jokipii, J.R.: 1990, in Physics of the Outer Heliosphere, Cospar Colloq. Ser. Vol. 1, Gzedzielski, S. and Page, D. E. (eds.), Pergamon, New York, pp. 169-178.

Jokipii, J. R., Levy, E. H. and Hubbard, W. B.: 1977, 'Effects of particle drift on cosmic ray transport I. General properties, application to Solar modulation', Astrophys. J. 213, 861.

Jokipii, J. R., and Thomas, B.T.: 1981, 'Effects of drifts on the transport of cosmic rays, IV, Modulations by a wavy interplanetary current sheet', Astrophys. J. 243, 1115-1122.

Jokipii, J.R. and Kóta, J.: 1989, 'The Polar Heliospheric Magnetic Field', Geophys. Res. Letters 16, $1-4$.

Jokipii, J. R. and Giacalone, J.: 1998, Space Science Rev., this volume.

Kóta, J., and Jokipii, J. R.: 1983, 'Effects of drifts on the transport of cosmic rays, VI, A three dimensional model including diffusion', Astrophys. J. 265, 573-581.

Kóta, J. and Jokipii, J. R.: 1991, 'The Role of Corotating Interaction Regions in Cosmic Ray Modulation', Geophys. Res. Lett. 18, 1797-1800.

Kóta, J. and Jokipii, J. R.: 1995, 'Corotating Variations of Cosmic Rays Near the South Heliospheric Pole', Science 268, 1024-1024.

Kóta, J. and Jokipii, J. R.: 1998, Space Science Rev., this volume.

Lazarus, A. J., Belcher, J. W., Paularena, K. I. and Richardson, J. D.: 1998, Space Science Rev., this volume.

Le Roux, J. A. and Potgieter, M. S.: 1995, 'The simulation of complete 11 and 22 year modulation cycles for cosmic rays in the heliosphere using a drift model with global merged interaction regions', Ap. J. 442, 847-851.

Le Roux, J. A., Potgieter, M. S. and Ptuskin, V. S.: 1996, 'A transport model for the diffusive shock acceleration of anomalous cosmic rays in the heliosphere', J. Geophys. Res. 101, 4791-4803.

Lockwood, J. A.: 1985, 'Solar Activity and Modulation of the Cosmic Ray Intensity', J. Geophys. Res. 90, 4439.

Lockwood, J. A. and Webber, W. R.: 1997, 'A comparison of cosmic ray intensities near the Earth at the sunspot minima in 1976 and 1987 and during 1995 and 1996', J. Geophys. Res. 102, $24,221-24,228$.

Lopate, C. and Simpson, J. A.: 1991, 'The physics of cosmic ray modulation: heliospheric propagation during the 1987 minimum', J. Geophys. Res. 96, 15,877-15,898.

McDonald, F. B., Moraal, H., Reinecke, J. P. L., Lal, N. and McGuire, R. H.: 1992, 'The cosmic radiation in the heliosphere at successive solar minima', J. Geophys. Res. 97, 1557-1570.

McDonald, F. B. and Lal, N.: 1995, 'The Initial Cosmic Ray Recovery Phase of Solar Cycle 22', Proc. 24th Int. Cosmic Ray Conf. (Rome), 4, 780-783.

McDonald, F.B., Ferrando, P., Heber, B., Kunow, H., McGuire, R., Müller-Mellin, R., Paizis, C., Raviart, A. and Wibberenz, G.: 1997, 'A Comparative Study of Cosmic Ray Radial and Latitudinal Gradients in the Inner and Outer Heliosphere', J. Geophys. Res. 102, 4643-4651.

McDonald, F. B.: 1998, Space Science Rev., this volume.

McKibben, R. B.: 1987, 'Galactic cosmic rays and anomalous components in the heliosphere', Review of Geophysics 25, 711-722.

McKibben, R.B.: 1989, 'Reanalysis and Confirmation of Positive Latitude Gradients for Anomalous Helium and Galactic Cosmic Rays Measured in 1975-1976 with Pioneer 11', J. Geophys. Res. 94, 17,021-17,033. 
McKibben, R.B., Connell, J. J., Lopate, C., Simpson, J. A. and Zhang, M.: 1996, 'Observations of Galactic Cosmic Rays and the Anomalous Helium during Ulysses Passage from the South to the North Solar Pole', Astron. Astrophys. 316, 547-554.

McKibben, R. B.: 1998, Space Science Rev., this volume.

Paizis, C., Heber, B., Raviart, A., Potgieter, M. S., Ferrando, P., and Müller-Mellin, R.: 1997, 'Compton-Getting Factor and Latitude Variation of Cosmic Rays', Proc. 25th Int. Cosmic Ray Conf. (Durban), 2, 93-96.

Parker, E. N.: 1965, 'The passage of energetic particles through interplanetary space', Planet. Space. Sci. 13, 9-49.

Phillips, J.L., Bame, S. J., Barnes, A., Barraclough, B. L., Feldman, W. C., Goldstein, B. E., Gosling, J. T., Hoogeveen, G. W., McComas, D. J., Neugebauer, M.and Suess, S. T.: 1995, 'Ulysses Solar Wind Plasma Observations from Pole to Pole', Geophys. Res. Lett. 22, 3301-3304.

Potgieter, M. S: 1993, 'Time-dependent modulation: role of drifts and interaction regions', Adv. Space Res. 13(6), 239-249.

Potgieter, M. S.: 1995, 'The Long-term modulation of galactic cosmic rays in the heliosphere', $A d v$. Space Res. 16(9): 191-203.

Potgieter, M. S.: 1997, 'The Heliospheric Modulation of Galactic Cosmic Rays at Solar Minimum', Adv. Sp. Res 19(6), 883-892.

Potgieter, M. S.: 1998, Space Science Rev., this volume.

Potgieter, M. S. and Moraal, H.: 1988, 'Acceleration of cosmic rays in the solar wind termination shock I, A steady state technique in a spherically symmetric model', Ap. J. 330, 445-455.

Potgieter, M. S., Le Roux, J. A. and Burger, R. A.: 1989, 'Interplanetary cosmic ray radial gradients with steady state modulation models', J. Geophys. Res. 94, 2323-2332.

Potgieter, M. S. and Le Roux, J. A.: 1994, Ap. J. 423, 817-827.

Potgieter, M.S., Haasbroek, L. J., Ferrando, P., and Heber, B.: 1997, 'The Modeling of the Latitude Dependence of Cosmic Ray Protons and Electrons in the Inner Heliosphere', Adv. Sp. Res 19(6), 917-920.

Rastoin, C., et al.: 1996, 'Time and space variations of the galactic cosmic ray electron spectrum in the 3-d heliosphere explored by ulysses', Astron. Astrophys. 307, 981-995.

Roelof, E. C., Simnett, G. M., Tappin, S. J.: 1996, 'The regular structure of shock-accelerated $\sim 40-100 \mathrm{keV}$ electrons in the high latitude heliosphere', Astron. Astrophys. 316, 481-486.

Sanderson, T. R., Bothmer, V., Marsden, R. G., Trattner, K. J., Wenzel, K.-P., Balogh, A., Forsyth, R. J., and Goldstein, B. E.: 1995, 'The Ulysses south polar pass: Energetic ion observations', Geophys. Res. Lett. 22, 3357-3360.

Simpson, J.A.: 1996, 'Ulysses Cosmic Ray Investigations Extending from the South to the North Polar Regions of the Sun and Heliosphere', Proc. 24th Int'l. Cosmic Ray Conf. (Rome), Il Nuovo Cimento, 19C, N.6, 935-943.

Simpson, J. A.: 1998, Space Science Rev., this volume.

Simpson, J. et al.: 1992, 'The ULYSSES cosmic-ray and solar particle investigation', Astron. Astrophys. Suppl. 92(2), 365-399.

Simpson, J.A., Anglin, J. D., Bothmer, V., Connell, J. J., Ferrando, P., Heber, B., Kunow, H., Lopate, C., Marsden, R. G., McKibben, R.B., Müller-Mellin, R., Paizis, C., Rastoin, C., Raviart, A., Sanderson, T. R., Sierks, H., Trattner, K. J., Wenzel, K.-P., Wibberenz, G. and Zhang, M.: 1995, 'Cosmic Ray and Solar Particle Investigations Over the South Polar Regions of the Sun', Science 268, 1019-1023.

Simpson, J.A., Zhang, M. and Bame, S.: 1996, 'A Solar Polar North-South Asymmetry for Cosmic Ray Propagation in the Heliosphere: The Ulysses Pole-to-Pole Rapid Transit', Ap. J. 465, L69L72.

Smith, E.J. and Balogh, A.: 1995, 'Ulysses Observations of the Radial Magnetic Field', Geophys. Res. Letters 22, 3317-3320.

Smith, E.J., Jokipii, J.R., and Kòta, J.: 1997, 'Modelling of Ulysses Pole-to-Pole Cosmic Ray Observations', Eos Trans. AGU 78(46), Fall Meet. Suppl., F547.

Steenberg, C. D. and Moraal, H.: 1995, 'Model calculations on anomalous hydrogen', Proc. 24th Int. Cosmic Ray Conf. (Rome), 4, 739-742. 
Steenberg, C. D. and Moraal, H.: 1996, 'An acceleration/modulation model for anomalous cosmic ray hydrogen in the heliosphere', Ap. J. 463, 776-783.

Steenberg, C. D. and Moraal, H.: 1997, 'An alternative approach to the modeling of anomalous cosmic ray intensities in the heliosphere', Adv. Space Res. 19(5), 805-808.

Steenkamp, R. and Moraal, H.: 1993a, 'Acceleration and modulation of anomalous helium and oxygen in 1977 and 1987', Proc. 23rd Int. Cosmic Ray Conf. (Calgary), 3, 419-422.

Steenkamp, R. and Moraal, H.: 1993b, 'Time-dependent and hysteresis effects in the modulation revisited', Proc. 23rd Int. Cosmic Ray Conf. (Calgary), 3, 539-542.

Steenkamp, R.: 1995, 'Shock acceleration as source of the anomalous component of Cosmic Rays in the Heliosphere', Ph.D. Thesis, Potchefstroom University for CHE, South Africa.

Stone, E. C.: 1987, 'Cosmic ray studies out of the ecliptic', Proc. 20th Int. Cosmic Ray Conf. (Moscow), 7, 105-114.

Stone, E. C., Cummings, A. C. and Webber, W. R.: 1996, 'The distance to the termination shock in 1993 and 1994 from observations of anomalous cosmic rays', J. Geophys. Res. 101, 11,01711,025 .

Suess, S.T., Smith, E. J., Phillips, J., Goldstein, B. E. and Nerney, S.: 1996, 'Latitudinal Dependence of the Radial IMF Component - Interplanetary Imprint', Astron. Astrophys. 316, 304-312.

Suess, S. T. et al.: 1998, Space Science Rev., this volume.

Thomas, B. T. and Gall, R.: 1984, 'Solar-Flare Induced Forbush Decreases: Dependence on Shock Wave Geometry', J. Geophys. Res. 89, 2991-2996.

Trattner, K.J., Marsden, R. G., Bothmer, V., Sanderson, T. R., Wenzel, K.-P., Klecker, B. and Hovestadt, D.: 1995, 'The Ulysses South Polar Pass: Anomalous Component of Cosmic Rays', Geophys. Res. Letters 22, 3349-3352.

Trattner, K.J., Marsden, R. G., Bothmer, V., Sanderson, T. R., Wenzel, K.-P., Klecker, B. and Hovestadt, D.: 1996, 'Ulysses COSPIN/LET: Latitudinal Gradients of Anomalous Cosmic Ray O, N, and Ne', Astron. Astrophys. 316, 519-527.

Venkatesan, D., Decker, R. B., and Krimigis, S. M.: 1984, 'Radial gradient of cosmic ray intensity from a comparative study of data from Voyager 1 and 2 and IMP 8', J. Geophys. Res. 89, $3735-3746$.

Webber, W. R. and Lockwood, J. A.: 1985, 'The cosmic ray interplanetary radial gradient from 1972-1985', Proc. 19th Int. Cosmic Ray Conf. (La Jolla), 5, 185-188.

Webber, W. R. and Lockwood, J. A.: 1997, 'Intensities of anomalous and galactic cosmic rays in the outer heliosphere near the heliospheric equator in 1987 and during the period from 1994 to 1996', J. Geophys. Res. 102, 9773-9779.

Webber, W. R. and Lockwood, J. A.: 1998, Space Science Rev., this volume.

Zhang, M., Simpson, J. A., McKibben, R. B., Johns, T. S., Smith, E. J., and Phillips, J. L.: 1995, 'Ulysses Observations of 26 Day Intensity Variations of Cosmic Rays and Anomalous Helium Over the South Pole', Proc. 24th Int. Cosmic Ray Conf. (Rome), 4, 956-959.

Zhang, M.: 1997, 'A linear relationship between the latitude gradient and 26 day recurrent variation in the fluxes of galactic cosmic rays and anomalous nuclear components. I. observations' Ap. $J$. 488, 841-853. 